\title{
Estudio electromiográfico, histoquímico y morfométrico de la musculatura paravertebral en escoliosis idiopática
}

IZQUIERDO NÚÑEZ, E.

\section{INTRODUCCIÓN}

En estos últimos años, han ido apareciendo publicaciones sobre la posible causa de la escoliosis idiopática, como consecuencia de alteraciones neuromusculares de la musculatura paravertebral.

Hay estudios electromiográficos que han demostrado un aumento de la actividad mioeléctrica de la musculatura paravertebral en el ápex de la convexidad de la curva y una falta de actividad mioeléctrica en el lado cóncavo a nivel del ápex (Lefebre y cols. ${ }^{47}$, Zuck y cols. ${ }^{17}$, Redfrod y cols. ${ }^{70}$, Zetterberg y cols. ${ }^{116}$, Schultz y cols. ${ }^{76}$, Reuber y cols. ${ }^{71}$, Donovan y cols. ${ }^{25}$, Trontelj y cols. ${ }^{91}$ ).

Estudios histoquímicos y morfométricos de fibras musculares en los músculos paravertebrales a nivel del ápex de la curva escoliótica, han encontrado distintos tipos de alteraciones: agrupamiento de fibras, contenido alto de mitocondrias y glucógeno, aumento del tejido conectivo, redondeamiento y variabilidad en la forma, vacuolas, cuerpos tubulares (Yaron y Robin, $109,110,11,112,113$ Sahgal y cols. ${ }^{72}$ ).

Aumento de fibras tipo I en el lado de la convexidad de la curva en relación a la concavidad, así como atrofia de fibras tipo I en el lado de la concavidad (Yaron y Robin $\left.{ }^{10,111,12}\right)$, o artrofia de fibras tipo II en el lado de la concavidad de la curva escoliótica (Sahgal y cols. ${ }^{72}$ ).

Estas alteraciones histopatológicas podían ser uno de los factores etiopatogénicos de la escoliosis idiopática según unos autores (Yaron y Robin, ${ }^{110,111,113}$ Sahgal y cols. ${ }^{72}$, Ovalle y Tredwell ${ }^{59}$, Jean Chen Chiu y cols. ${ }^{41}$, Bylund y cols. ${ }^{17}$ ), mientras que otros autores (Zetterberg y cols. ${ }^{1 / 7}$, Ford y cols. ${ }^{28,29}$, Hsu y cols. ${ }^{39}$, Gibson y cols. ${ }^{30}$ ) opinan que estas alteraciones histopatológicas eran consecuencia de una adaptación de las fibras musculares a la curva escoliótica.

El objetivo de este trabajo ha sido estudiar la musculatura paravertebral a nivel del ápex de la curva de enfermos que presentan escoliosis idiopática desde el punto de vista electromiográfico, e histoquímico y morfométrico, y valorar si pudiera existir alguna interrelación entre los hallazgos electromiográficos e histoquímicos y morfométricos.

\section{MATERIAL Y MÉTODO \\ MATERIAL PARA EL ESTUDIO ELECTROMIOGRÁFICO}

Consta de 24 enfermos. Se registró la actividad electromiográfica de la musculatura paravertebral torácica y lumbar en los músculos longíssimo y multifido del ápex de sendas curvas de la convexidad y concavidad en las posiciones de decúbito y bipedestación y en actitudes de reposo y contra resistencia.

Las mediciones de las curvas que presentan esto 24 enfermos eran las siguientes:

\begin{tabular}{lcccc}
\hline $\begin{array}{l}\text { Tipo de } \\
\text { curva }\end{array}$ & $\begin{array}{c}\text { N. } \\
\text { enfermos }\end{array}$ & Edad & $\begin{array}{c}\text { Curva } \\
\text { torácica }\end{array}$ & $\begin{array}{c}\text { Curva } \\
\text { lumbar }\end{array}$ \\
\hline King I & 4 & 15 & $54.7^{\circ}$ & $69.7^{\circ}$ \\
King II & 13 & 16 & $58.1^{\circ}$ & $37.2^{\circ}$ \\
King III & 1 & 15 & $43.0^{\circ}$ & $14.0^{\circ}$ \\
King V & 1 & 18 & $84.0^{\circ}$ & $98.0^{\circ}$ \\
Lumbar & 5 & 17 & $32.6^{\circ}$ & $47.8^{\circ}$ \\
\hline
\end{tabular}

En 17 enfermos se practicó una $2^{\underline{a}}$ electromiografía en el músculo longissimo a los 6 meses del post-operatorio. No se practicó la electromiografía del músculo multífico para no contactar la aguja del electrodo con la instrumentación y evitar riesgo de infección. Las curvas según la clasificación de King fueron:

$\begin{array}{ll}\text { King I: } & 4 \\ \text { King II: } & 7 \\ \text { King III: } & 1 \\ \text { King V: } & 1 \\ \text { Lumbar: } & 4\end{array}$

Las mediciones de las correcciones post-operatorias obtenidas en estas curvas fueron las siguientes:

Curva King I: (4). Edad media 15 años.

\begin{tabular}{lccc}
\hline Curva & Pre-operat. & Post-operat. & \% Corrección \\
\hline Torácica & $54.7^{\circ}$ & $23.2^{\circ}$ & 57.5 \\
Lumbar & $69.7^{\circ}$ & $26.7^{\circ}$ & 61.7 \\
\hline
\end{tabular}

En dos casos se instumentó la curva lumbar con técnica de Zielke por vía anterior, y en un segundo tiempo con instrumentación Cotrel-Dubousset por vía posterior la curva torácica y lumbar. 
Tipo King II: (7). Edad: 16 años y 3 meses

\begin{tabular}{lccc}
\hline Curva & Pre-operat. & Post-operat. & \% Corrección \\
\hline Torácica & $58.14^{\circ}$ & $24.79^{\circ}$ & 57.37 \\
Lumbar & $37.21^{\circ}$ & $19.93^{\circ}$ & 46.44 \\
\hline
\end{tabular}

La curva instrumentada fue la curva torácica con técnica Cotrel-Dubousset, siendo la corrección de la curva lumbar espontánea.

Curva King III: (1). Edad: 15 años

\begin{tabular}{lccc}
\hline Curva & Pre-operat. & Post-operat. & \% Corrección \\
\hline Torácica & $43^{\circ}$ & $4^{\circ}$ & 90.69 \\
Lumbar & $14^{\circ}$ & $0^{\circ}$ & 100.00 \\
\hline
\end{tabular}

Solo se instrumentó la curva torácica con la técnica Cotrel-Dubousset. La curva lumbar practicamente inexistente corrigió un $100 \%$ espontáneamente.

Tipo King V: (1). Edad: 18 años

\begin{tabular}{lccc}
\hline Curva & Pre-operat. & Post-operat. & \% Corrección \\
\hline Torácica & $84^{\circ}$ & $48^{\circ}$ & 42.85 \\
Lumbar & $98^{\circ}$ & $50^{\circ}$ & 48.97 \\
\hline
\end{tabular}

Se practicó fusión anterior de la curva toraco-lumbar y en un $2^{\circ}$ tiempo se fusionarán ambas curvas por vía posterior e instrumentación Cotrel-Dubousset.

Curva Lumbar: (4). Edad: 17 años y 2 meses

\begin{tabular}{lccc}
\hline Curva & Pre-operat. & Post-operat. & \% Corrección \\
\hline Torácica & $32.6^{\circ}$ & $15.4^{\circ}$ & 52.76 \\
Lumbar & $47.8^{\circ}$ & $7.6^{\circ}$ & 84.10 \\
\hline
\end{tabular}

Se intervino la curva lumbar con instrumentación de Zielke, siendo la corrección de la curva torácica espontánea.

\section{MATERIAL PARA EL ESTUDIO HISTOQUÍMICO Y MORFOMÉTRICO}

Ha constado de biopsias de músculos paravertebrales longíssimo y multífido de ambos lados de la curva, y glúteo del lado derecho en 15 enfermos que presentaban escoliosis idiopática torácica de convexidad derecha y lumbar izquierda, siendo en 13 casos la curva tipo King II, en 1 caso tipo King I y en 1 caso tipo King III.

Los 15 enfermos estudiados correspondieron a 10 mujeres y 5 varones, con una edad máxima de 29 años y mínima de 12 , con una media de 16,4 años. La medición de la curva torácica era de $68^{\circ}$ con un máximo de $135^{\circ}$ y mínimo de $43^{\circ}$.

Estudio histoquímico y morfométrico en 15 de estos 24 enfermos de músculos longíssimo y multífido a nivel del ápex de la curva escoliótica en la convexidad y concavidad de la curva torácica.

\begin{tabular}{lcccc}
\hline $\begin{array}{l}\text { Tipo de } \\
\text { curva }\end{array}$ & $\begin{array}{c}\text { N. } \\
\text { enfermos }\end{array}$ & Edad & $\begin{array}{c}\text { Curva } \\
\text { torácica }\end{array}$ & $\begin{array}{c}\text { Curva } \\
\text { lumbar }\end{array}$ \\
\hline King I & 1 & 12 & $74.0^{\circ}$ & $80.0^{\circ}$ \\
King II & 13 & 18 & $71.6^{\circ}$ & $40.7^{\circ}$ \\
King III & 1 & 15 & $43.0^{\circ}$ & $14.0^{\circ}$ \\
\hline
\end{tabular}

\section{MÉTODO Y TÉCNICA}

\section{OBTENCIÓN DE LOS REGISTROS ELECTROMIOGRÁFICOS}

La relación de este apartado la dividiremos, debido a su complejidad, en diferentes puntos:
a) Electromiógrafo.
b) Electrodos.
c) Proceso de registro.
d) Músculos explorados y posiciones.

\section{a) Electromiógrafo}

Fue utilizado para el estudio de la actividad muscular un aparato Medelec con capacidad de muestra de 2 canales con módulos totalmente intercambiables, un ancho rango de aplicaciones con un sistema de registro de papel revelado a la luz y vista y registro simultáneo.

Utiliza el aparato Medelec un amplificador principal AA - GMK. II - B para amplificar señales biológicas procedentes del paciente a través de los electrodos.

El pre-amplificador está conectado al amplificador principal por un cable flexible muy corto e incorpora las conexiones para los electrodos al paciente evitando interferencias.

Se ha tenido siempre en cuenta de poner la misma calibración en ambos canales para que los registros de las unidades motoras y de los potenciales voluntarios a ambos lados de la curva respondan a los mismos parámetros. La visualización se realiza sobre el osciloscopio del electromiográfo y posteriormente se pasaba a registrar un papel fotográfico para una posterior medición. Incorpora un amplificador de audio con selector de señal para vigilancia aural usando un altavoz externo.

\section{b) Electrodos}

La actividad eléctrica muscular fue captada por electrodos de aguja autoclavables. Consiste en un cable de platino/iridium localizado dentro de una cánula de acero inoxidable y embutido en araldite.

La conexión entre la aguja y el cable asegura una rápida conexión eléctrica libre de fallos y los conectores de oro plateado aseguran un buen contacto.

La punta del electrodo tiene un ángulo de $15^{\circ}$. El área de la punta es de $0,07 \mathrm{~mm} .^{2}$ 


\section{c) Proceso de registro}

El desarrollo de una alta frecuencia y alta definición en el sistema de registro hacen posible obtener un trazado de registro directo sobre papel Linagraph, por el uso de un tubo catódico de fibra de vidrio.

El papel es sensible a los rayos ultravioleta en contacto con la película bajo la fibra de vidrio.

\section{d) Músculos explorados y posiciones:}

Se obtuvieron los registros de los siguientes músculos:

Longíssimo de la convexidad de la curva torácica.

Longissimo de la concavidad de la curva torácica.

Longíssimo de la convexidad de la curva lumbar.

Longíssimo de la concavidad de la curva lumbar.

Multífido de la convexidad de la curva torácica.

Multífido de la concavidad de la curva torácica.

Multífido de la convexidad de la curva lumbar.

Multífido de la concavidad de la curva lumbar.

\section{Cada músculo fue explorado independientemente en:}

a) Reposo. Con el sujeto en decúbito prono con los miembros superiores a lo largo del tronco y bien relajado para descartar potenciales extraños o patológicos a la exploración.

b) Decúbito prono contra resistencia.' El sujeto realizaba un ejercicio de hiperextensión contra la resistencia que le oponía el explorador con la palma de la mano situado a nivel de las espinosas C7-T1.

\section{c) Bipedestación y descalzo con el sujeto en reposo.}

d) En bipedestación y descalzo contra resistencia. $\mathrm{El}$ sujeto realizaba un ejercicio de hiperextensión contra la resistencia que le oponía el explorador con la palma de la mano situada a nivel de las espinosas de $\mathrm{C}_{7}$. T1.

Todos los registros se fijaron en un mapa anatómico donde se dibujaba la curva y se señalaba el nivel y localización del músculo explorado. Los electrodos se situaron en el vientre muscular de cada uno de los músculos. El músculo multífido se pinchó a nivel de ambas partes del ápex de la curva torácica y lumbar a 3 centímetros de la apófisis espinosa y dirigiendo el electrodo profundamente y en dirección oblicua hacia medial hasta contactar con la unión de la espinosa con la lámina. El músculo longíssimo se pinchó a nivel de ambas partes del ápex de la curva torácica y lumbar a unos 6 centímetros de la apófisis espinosa y dirigiendo en profundidad el electrodo a unos 4 centímetros.

Una vez colocado el electrodo en el vientre muscular deseado se incitó al sujeto a realizar movimientos de hiperextensión de columna observando el registro por Scopia, a la vez que mediante el sonido nos guiábamos de la situación de la placa motora de fácil localización por su característico golpeteo, comparado a una pieza de ametralladora con un ruido corto, alternante y seco que se modifica en intensidad y frecuencia cuando el enfermo procede a aumentar la función muscular que se explora.
Así pues y sólo cuando existía la certeza de tener localizado el músculo y un grupo de placas motoras en condiciones perfectas se procedía a someter al sujeto a la exploración experimental en decúbito y bipedestación y en estas dos posiciones en reposo y contra resistencia. El estudio no pudo ser completo al sufrir algunos enfermos dolor y mareo al no soportar la aguja del electrodo.

El ejercicio contra resistencia siempre se palicaba por el mismo explorador y situando la mano sobre las espinosas $\mathrm{C}_{7} \mathrm{Tl}$ y el enfermo empujaba en un movimiento de hiperextensión de columna contra dicha mano.

Los registros electromiográficos obtenidos se clasificaron siguiendo la clasificación de Buchthal y Clemensen, tal y como expusieron en su trabajo «On the differentiation of muscle atrophy by electromyography», en 1941.

Según estos autores, la contracción muscular registrada en el electromiógrafo puede ser:

a) Contracción nula. Correspondiente a aquellos registros que no presentan alteración en la línea de base. Se le dio valor 0 .

b) Contracción débil. La que aparece en aquellos trazados que presentan alteraciones de la línea base, muy específicas individualizadas y fácilmente reconcibles. Se le dio valor 1 .

c) Contracción moderada. En la que el trazado se ve enriquecido por nuevas unidades motoras, que frecuentemente se superponen, existiendo por lo general, una mayor amplitud del registro. Se le dio valor 2 .

d) Contracción fuerte. En la que ya aparece una pérdida de la línea base del registro que nos permite diferenciar las distintas unidades motoras y que normalmente se ve acompañada de un ritmo interferencial. Se le dieron valores 3-4 según la intensidad.

Obtención de los músculos para el estudio histoquímico y morfométrico.

Durante la intervención quirúrgica por vía pósterior, se obtuvieron muestras de músculos multífido y longíssimo a ambos lados del ápex de la curva torácica y del músculo glúteo mayor del lado de la convexidad de la curva torácica aprovechando en este caso que era el sistio para extraer injerto de la cresta ilíaca.

Los músculos se etiquetaron en 5 recipientes y se enviaron con la mayor urgencia posible al departamento de ANATOMIA PATOLÓGICA DEL HOSPITAL $1^{\circ}$ DE OCTUBRE, desde el HOSPITAL CENTRAL DE LA CRUZ ROJA. Envueltos en una gasa húmeda de suero fisiológico. Nunca transcurrió más de una hora en este traslado para evitar que se deteriorasen las muestras.

Cada músculo fué colocado por separado en una platina y congelado en isopentano previamente enfriado en nitrógeno líquido. Posteriormente se realizaron cortes transversales de 8 micras de grosor por medio de un criostato sobre las que se llevaron a cabo las siguientes 
técnicas histológicas e histoquímicas: Hematoxilinaeosina, PAS, Sudan Rojo, ATPasa preincubada a PH $9,4,4,6$ y 4,3 , DPNH y SDH (figuras 1 y 2 ).

El estudio morfométrico se realizó sobre cortes teñidos con ATPasa por medio de un Analizador de Imágenes semiautomático KONTRON, MOP Videoplan. Se efectuó un recuento de al menos 250 fibras por cada músculo.

\section{RESULTADOS DE LOS ESTUDIOS ELECTROMIOGRÁFICOS}

Los resultados electromiográficos obtenidos en cada uno de los músculos estudiados en las distintas posiciones y actitudes y partiendo de la ya clásica clasificación de Buchtal y Clemensen, se han clasificado en los cuatro grados ya conocidos de contracción muscular.

A los trazados electromiográficos nulos se les dió el valor 0 , a los debiles el valor 1 , a los moderados el valor 2 y a los fuertes se les dió el valor 3 ó 4 dependiendo del mayor ó menor trazado. De esta manera convertimos en valor numérico la interpretación de la respuesta electromiográfica.

Los valores obtenidos en los músculos analizados longíssimo y multífido en las posiciones de decúbito y bipedestación y en las actitudes de reposo y contra resistencia se exponen en la tabla ${ }^{\circ} 1$.

Análisis del conjunto del comportamiento muscular

\section{Pre-operatorio torácica decúbito reposo (gráfico 1):}

Se observa una mayor actividad electromiográfica en ambos músculos longissimo y multifido en el lado convexo en relación al cóncavo principalmente en las curvas $\mathrm{K} 2$, es decir en las curvas dobles donde la curva torácica es mayor que la curva lumbar.

\section{Post-operatorio torácica decúbito reposo} (gráfico 2):

Se observa la gran disminución de la actividad electromiógrafica en el lado convexo en relación al obtenido en el preoperatorio.

\section{Pre-operatorio torácica bipedestación reposo (gráfico 3):}

Igualmente que en la posición de decúbito se observa una mayor actividad electromiográfica en el lado convexo que en el cóncavo más evidente en el músculo longissimo que en el músculo multífido.

\section{Post-operatorio torácica bipedestación reposo (gráfico 4):}

Se observa una desaparición total de la actividad en el lado convexo en todos los tipos de curvas, excepto en un caso de curva tipo K5, que persistió actividad valor 2 en el lado convexo (preoperatorio valor 3). En el lado cóncavo actividad valor 1 en este mismo caso y en un caso de curva lumbar.

\section{Pre-operatorio lumbar decúbito reposo (gráfico 5):}

Existe actividad en el músculo longíssimo del lado convexo en las curvas tipo $\mathrm{K} 1, \mathrm{~K} 2, \mathrm{~K} 5$ y L. No hay actividad en el lado cóncavo en ningún tipo de curva en ambos músculos.

Los registros obtenidos a nivel del músculo multífido en el lado convexo, en la misma posición y actitud demuestran que existe actividad en las curvas tipo K1, K2 y K5, no observándose actividad en la curva tipo K3 y L. En el lado cóncavo no aparece ningun tipo de actividad en ninguna curva.

\section{Post-operatorio lumbar decúbito reposo (gráfico 6):}

Desapareció la actividad electromiográfica registrada en el músculo longíssimo del lado convexo, persistiendo la falta de actividad en el lado cóncavo.

\section{Pre-operatorio lumbar bipedestación reposo (gráfico 7):}

Se observa actividad en las curvas tipo K1 y L., a nivel del músculo longíssimo en el lado convexo. En el lado cóncavo no apareció actividad en ninguna curva.

En el músculo multífido se observa aumento la actividad en el lado convexo en las curvas tipo K1, K2, K5 y L. En lado cóncavo se observa aumento de actividad en el único caso de curva tipo K5 pero menos intensidad que en el lado convexo.

\section{Post-operatorio lumbar bipedestación reposo (gráfico 8):}

Hay una disminución de la actividad en el lado convexo en las curvas tipo $\mathrm{K} 1$ y L, pero aparece actividad en un caso de curva tipo $\mathrm{K} 2$, tanto en el lado convexo como en el lado cóncavo.

\section{RESULTADOS DEL ESTUDIO HISTOQUÍMICO}

El estudio con microscopio óptico demostró los siguientes hallazgos:

a) Aumento del tejido conectivo endomisial en tres enfermos en músculos aislados.

b) Fibras atróficas con las características de la atrofia por denervación en el músculo longíssimo de la concavidad de dos casos.

c) Fibras con abundante glucógeno y lípidos en músculos de siete casos.

d) Zonas con ausencia de tinción con los enzimas oxidativos, generalmente grandes y únicas de aspecto muy parecido a los cores, afectando a las fibras tipo I de los músculos longíssimo de la convexidad en seis casos y en músculos multífido de la convexidad en cinco casos, músculos multífidos de la concavidad de cuatro casos y longíssimos de la concavidad en un caso. (figs. 3 y 4). 

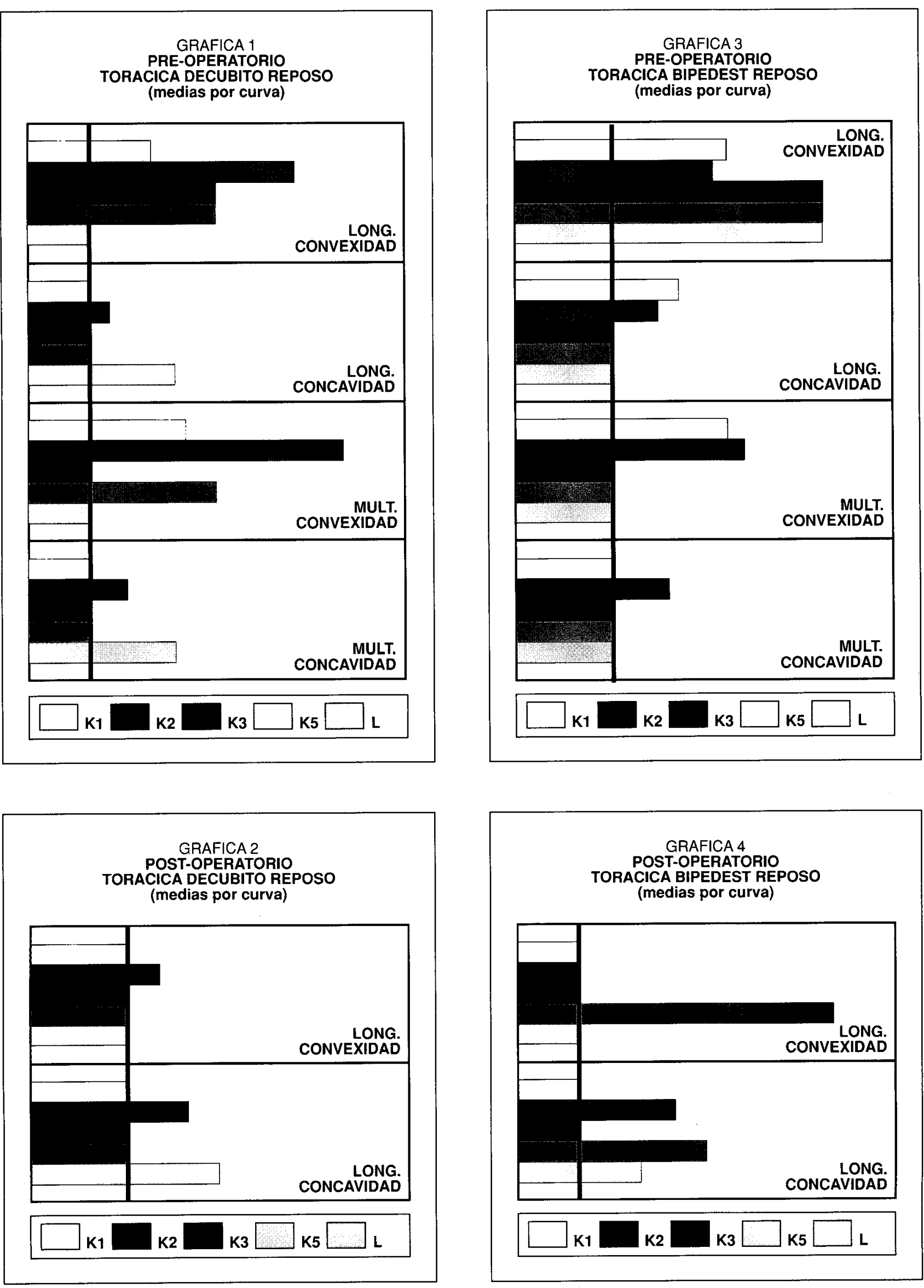


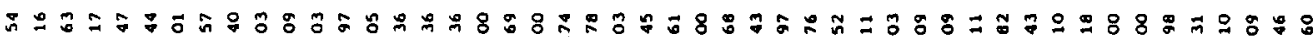

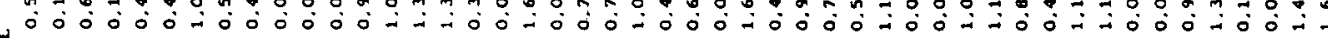

888888888888888888888888888888888888888888888888

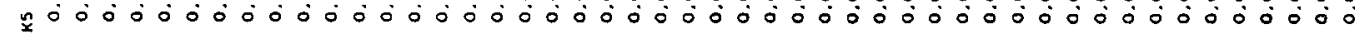

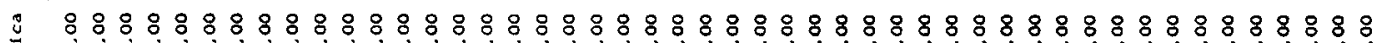
قي ⿷匚

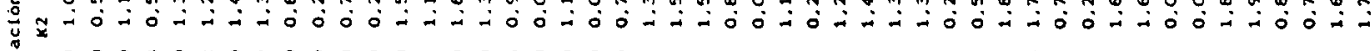

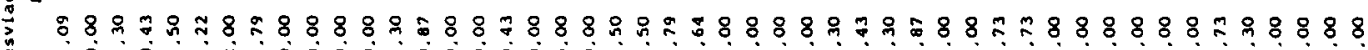
ý

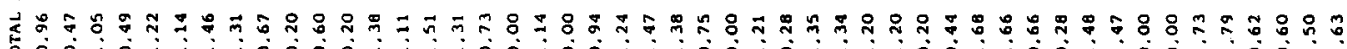

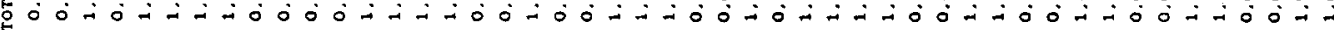

$=m:$ is 88888

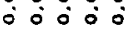
88.88

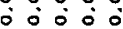
$\because \div 8:$ $\dot{m} \dot{\sigma} \dot{\sigma} \dot{\sigma}$ $\simeq$ 象和里

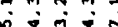

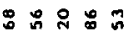
$\dot{2} \cong$

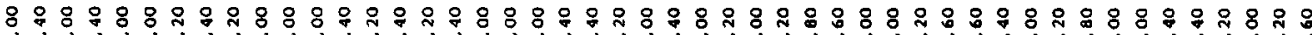

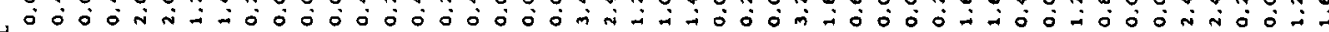
ᄂ 88888888888888888888888888888888888888888888888

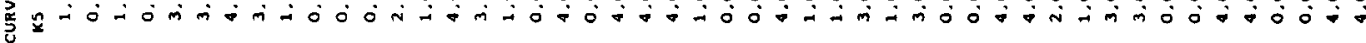
范

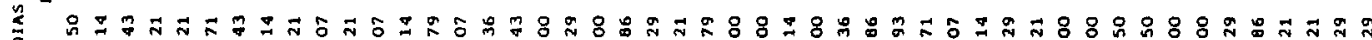

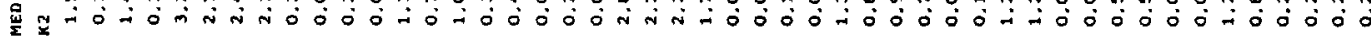

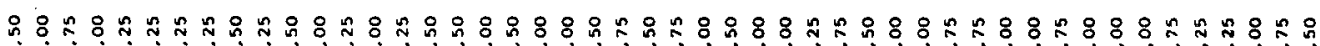

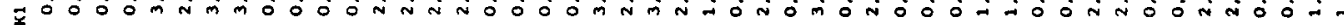

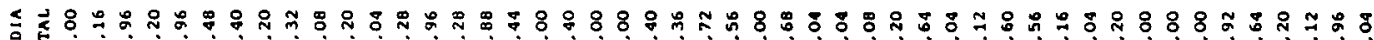

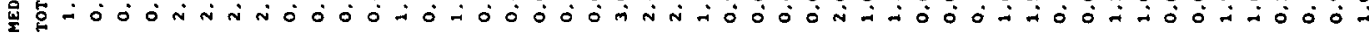
ñ $0000 \ldots+\infty$ n 7 j

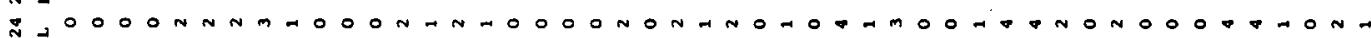

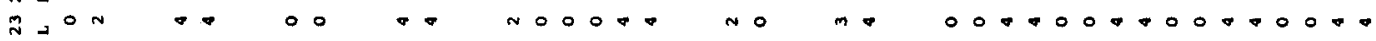
N a

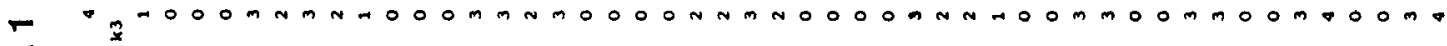

4 i $x_{-0}-0 m m m m$

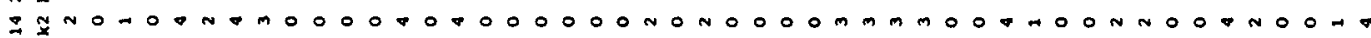

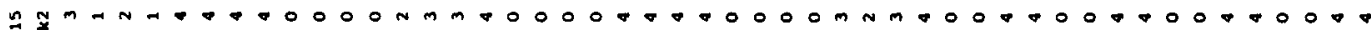

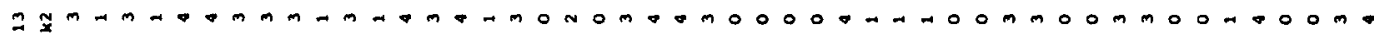

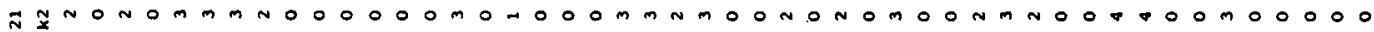
舟

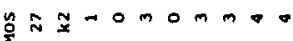

$0000 \rightarrow \infty$

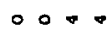

$\circ \circ \rightarrow$

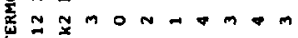

$0000 \%+\infty$

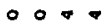

$\circ \circ \cdot m$

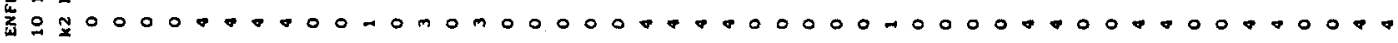

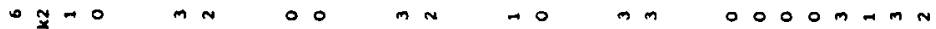

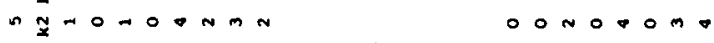

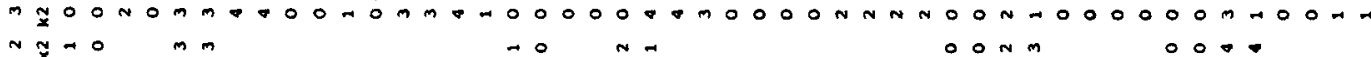

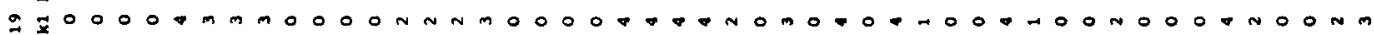
I $\vec{z}$ m

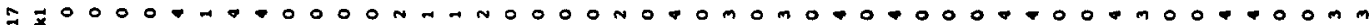

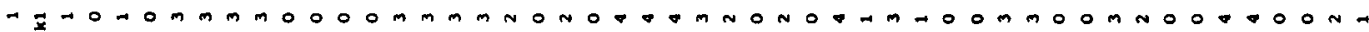

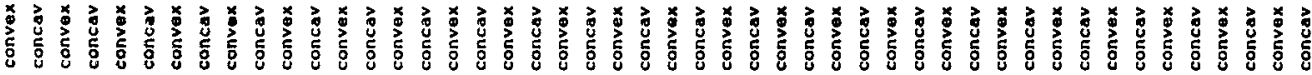
等

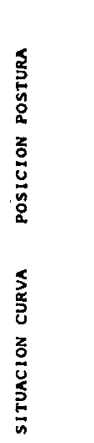

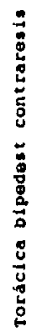

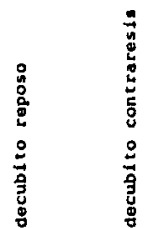
$\stackrel{5}{3}$

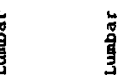<smiles>[CH][CH]</smiles>

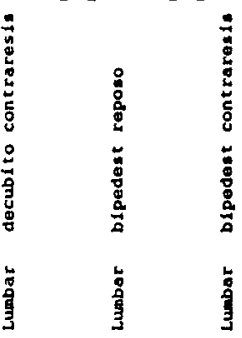

:

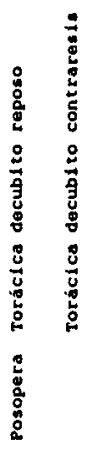

:

임

兽

孛

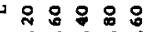

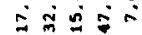

×88888 $\because \div$

ธะ8888 ㅎำ

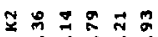

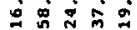

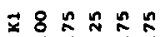

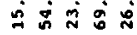

중 品品

工 $\approx$ ก

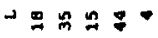

$\sim \cong 900$

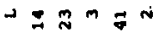

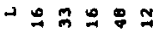

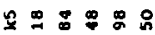
$x \approx 7 \div 0$

$\therefore \approx$ 은 웅

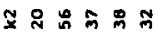
도ำ ×

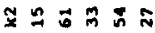
呦

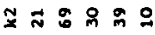

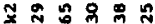
บำ

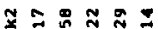

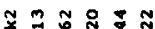

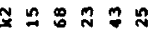

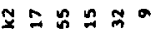

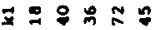
픔요

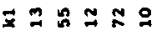
픔ㅇㅇㅇㅇㅇ 

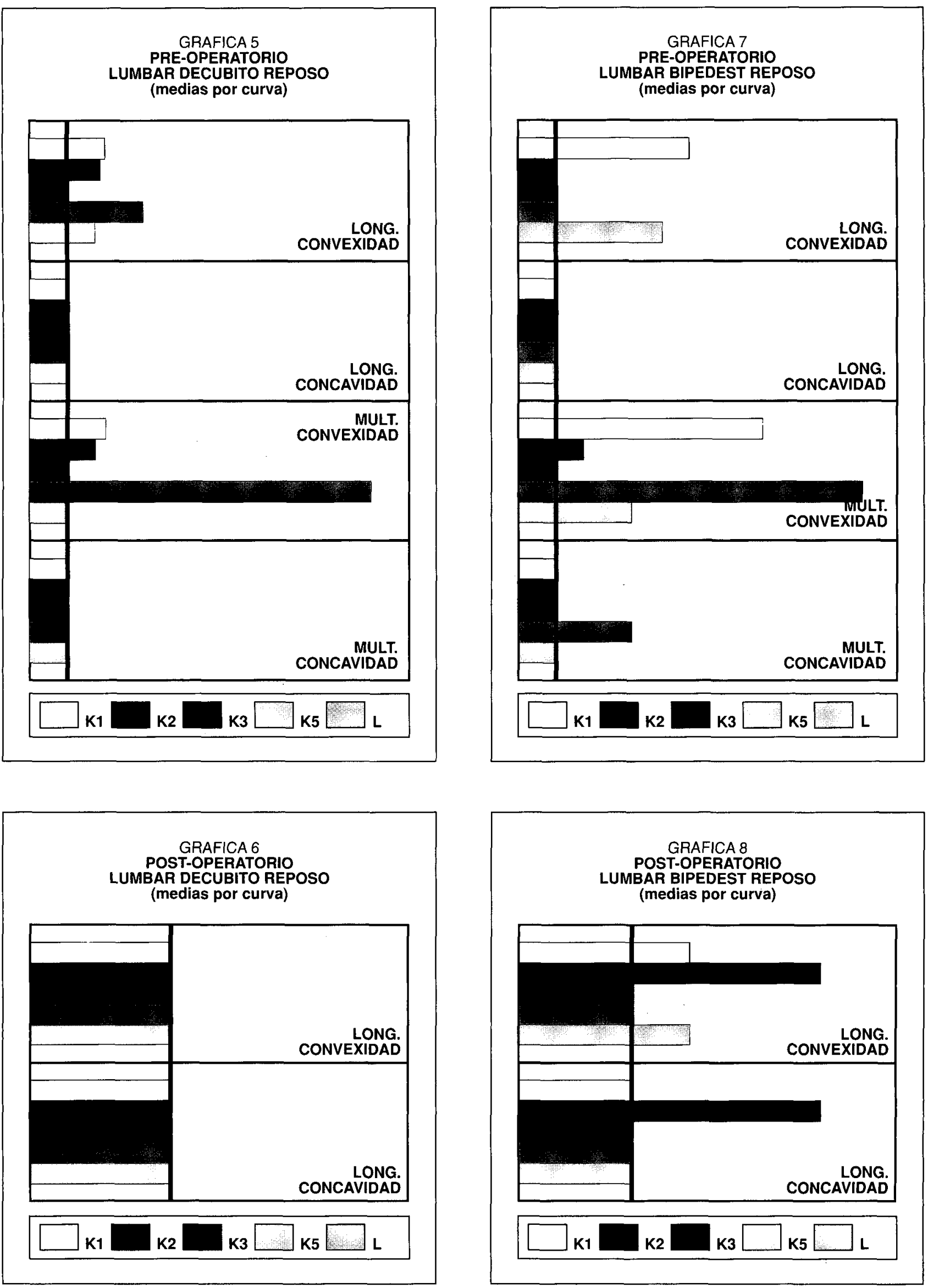


\section{TABLA 2}

\begin{tabular}{|c|c|c|c|c|c|c|c|c|c|c|c|c|c|c|c|c|c|c|}
\hline & Identif & 870322 & $871263^{\circ}$ & $865565^{3}$ & 71184 & $871154^{3}$ & 871505 & 871205 & $\begin{array}{r}8 \\
892636\end{array}$ & $\begin{array}{r}10 \\
899111\end{array}$ & $\begin{array}{r}11 \\
885652\end{array}$ & $89876^{\prime}$ & $\begin{array}{r}13 \\
01032\end{array}$ & $\begin{array}{r}1 \\
90249\end{array}$ & 9029 & & EIA & \\
\hline Long. convexidad & $\begin{array}{l}\text { TIPO I } \\
\text { TIPO II }\end{array}$ & $\begin{array}{l}200 \\
122\end{array}$ & $\begin{array}{l}155 \\
162\end{array}$ & 209 & 113 & 180 & 167 & 172 & 113 & 145 & 211 & 164 & 111 & 129 & 199 & 137? & 160.3 & 33.9 \\
\hline Long concavidad & TIPO 1 & 120 & $\begin{array}{l}117 \\
117\end{array}$ & $\begin{array}{l}84 \\
84\end{array}$ & 185 & & $\begin{array}{c}42 \\
109\end{array}$ & $\begin{array}{l}100 \\
124\end{array}$ & 111 & $\operatorname{lis}_{148}^{11}$ & $\begin{array}{l}158 \\
108\end{array}$ & $\begin{array}{r}84 \\
121\end{array}$ & $\begin{array}{l}207 \\
103\end{array}$ & 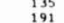 & & $\begin{array}{r}86 \\
116\end{array}$ & $\begin{array}{l}118.4 \\
1288.2\end{array}$ & $\begin{array}{l}35.7 \\
29.7\end{array}$ \\
\hline & TIPO is & 93 & 114 & 58 & 89 & & 105 & 132 & & 100 & 52 & 124 & 200 & 77 & $\begin{array}{l}124 \\
120\end{array}$ & $\begin{array}{l}116 \\
97\end{array}$ & $\begin{array}{l}128.2 \\
105.0\end{array}$ & 36.0 \\
\hline Mule, Convexidad & TIPO 1 & 116 & 97 & 148 & 137 & & 137 & 142 & 148 & 133 & 165 & 205 & 87 & & 182 & & 141.4 & $\begin{array}{l}36.0 \\
31.7\end{array}$ \\
\hline mit concaysdad & TIPo 11 & 234 & $\begin{array}{l}70 \\
68\end{array}$ & 299 & 113 & & 84 & 94 & 114 & 99 & 115 & 70 & 98 & 93 & 82 & & 105.0 & 59.9 \\
\hline mere concaviasa & TIPO if & $\begin{array}{l}227 \\
107\end{array}$ & $\begin{array}{l}68 \\
53\end{array}$ & $\begin{array}{l}183 \\
188\end{array}$ & 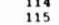 & & $\begin{array}{r}121 \\
99\end{array}$ & 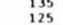 & & $\begin{array}{l}102 \\
118\end{array}$ & $\begin{array}{r}79 \\
169\end{array}$ & $\begin{array}{l}156 \\
116\end{array}$ & $\begin{array}{l}173 \\
177 \\
177-30\end{array}$ & $\begin{array}{r}158 \\
97\end{array}$ & $\begin{array}{l}122 \\
108\end{array}$ & $\begin{array}{c}157 \\
95\end{array}$ & $\begin{array}{r}136.5 \\
1120.5\end{array}$ & $\begin{array}{l}40.8 \\
35,9\end{array}$ \\
\hline Gluteo & $\begin{array}{l}\text { T1PO I } \\
\text { TPO }\end{array}$ & & 145 & 126 & 183 & & 102 & 192 & 125 & 121 & 128 & 192 & 206 & 145 & is 1 & 152 & 151.4 & $\begin{array}{l}31,1 \\
31,1\end{array}$ \\
\hline & & & & 116 & & & 111 & 102 & 116 & 100 & 102 & 52 & 104 & 85 & 109 & 85 & 97.5 & 16,7 \\
\hline
\end{tabular}

\begin{tabular}{|c|c|c|c|c|c|c|c|c|c|c|c|c|c|c|c|c|c|c|}
\hline & & & & & & & & & & & & & & & & & & \\
\hline Lang convexidad & & & & & & & & & & & & & & & & & & \\
\hline concavidad & & 39.7 & & 60,3 & 46,8 & 25.4 & 23.3 & 38.8 & 37.9 & 50.5 & 30.3 & & 40.3 & 33,0 & 39.9 & 42.8 & 39.1 & \\
\hline Long. Concavidad & $\begin{array}{l}\text { TIPO } \\
\text { TIPO }\end{array}$ & $\begin{array}{l}33.2 \\
33.6\end{array}$ & $\begin{array}{l}27.3 \\
23.3\end{array}$ & & $\begin{array}{l}38.8 \\
39.4\end{array}$ & & 27.5 & 26.4 & & ${ }_{37}^{41}$. & 48.6 & 53.6 & 38.0 & 43,1 & 32,8 & 41.9 & 37.7 & 8 \\
\hline convexidad & $\mathrm{t}$ & $\begin{array}{r}39,4 \\
\end{array}$ & $\begin{array}{l}31.9 \\
31.9\end{array}$ & & $\begin{array}{l}30.4 \\
40.1\end{array}$ & & 37 & & & & & 57 & $\begin{array}{l}31.5 \\
57.8\end{array}$ & & $\begin{array}{l}39,1 \\
35,2\end{array}$ & & & \\
\hline & IPO is & 36.8 & 29.9 & 37.4 & 44. & & 36 & 40.4 & 52.6 & & & & & & & & & H \\
\hline mile. Concavidad & TIP & 36.0 & 22.5 & 38,8 & 43. & & 29 & 28.6 & & & & & & & & & & \\
\hline Gluteo & & & & $\begin{array}{l}35.1 \\
38.0\end{array}$ & & & & & & & & & & & & & & \\
\hline Gereo & TIPO HI & & & $\begin{array}{l}38,0 \\
46,3\end{array}$ & 33.4 & & & & & & & & 43.6 $>3$ & & & 37. 0 & 8 & \\
\hline
\end{tabular}

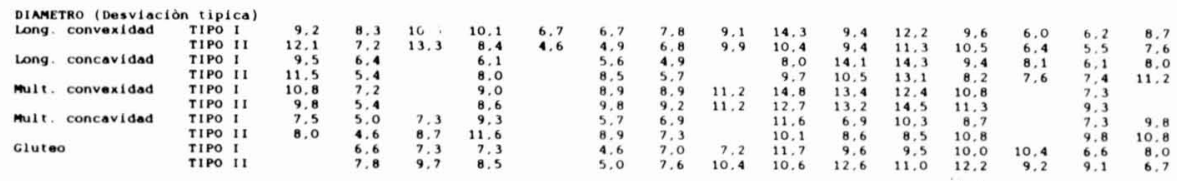

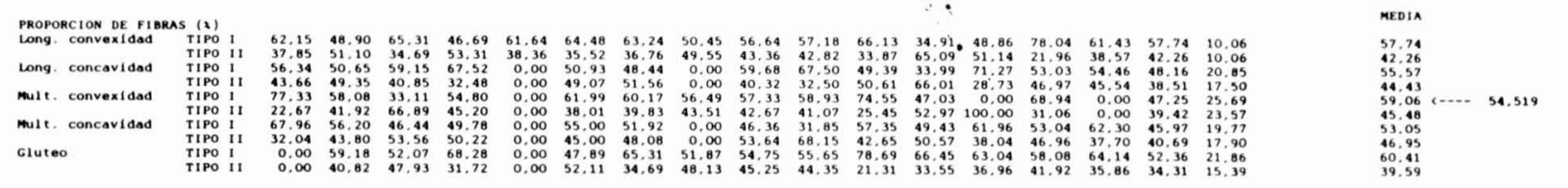

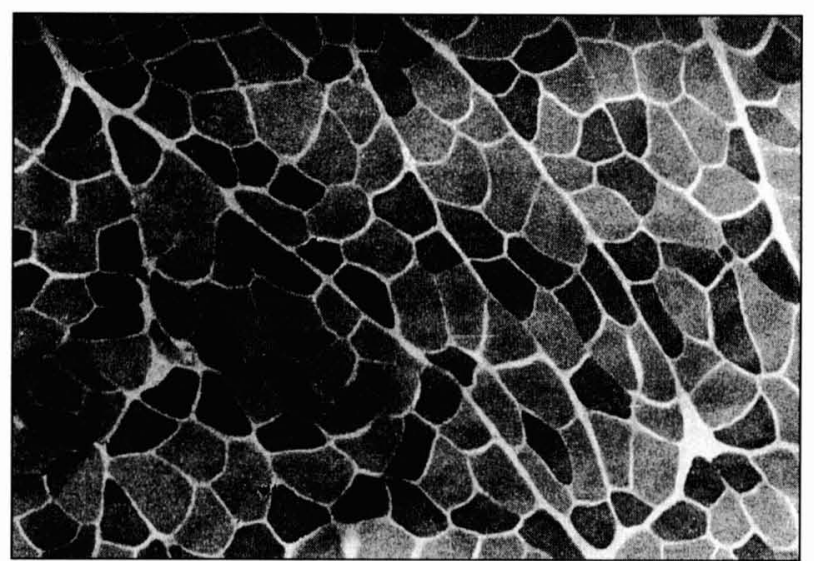

Figura 1. Variabilidad en el diámetro de las fibras debido a atrofia moderada de fibras tipo II (ATPasa pH 9.4 x 135)

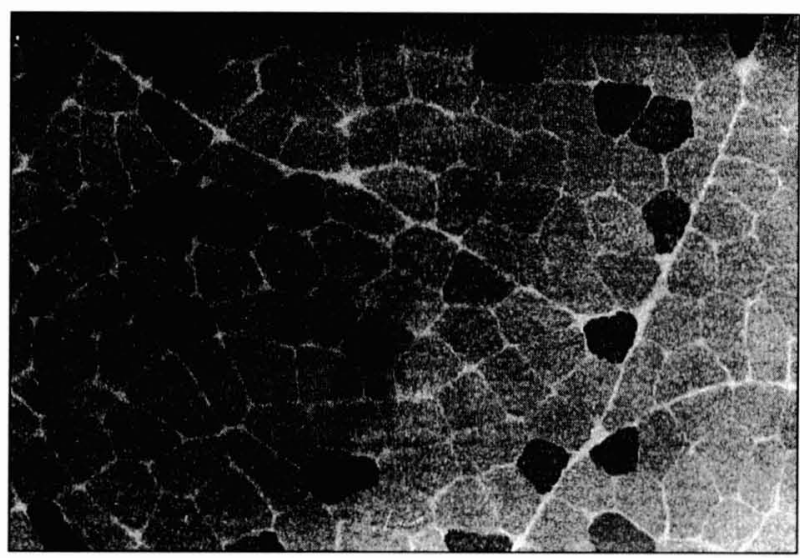

Figura 2. Gran predominio de fibras tipo I (ATPasa pH 9.4 x 135).

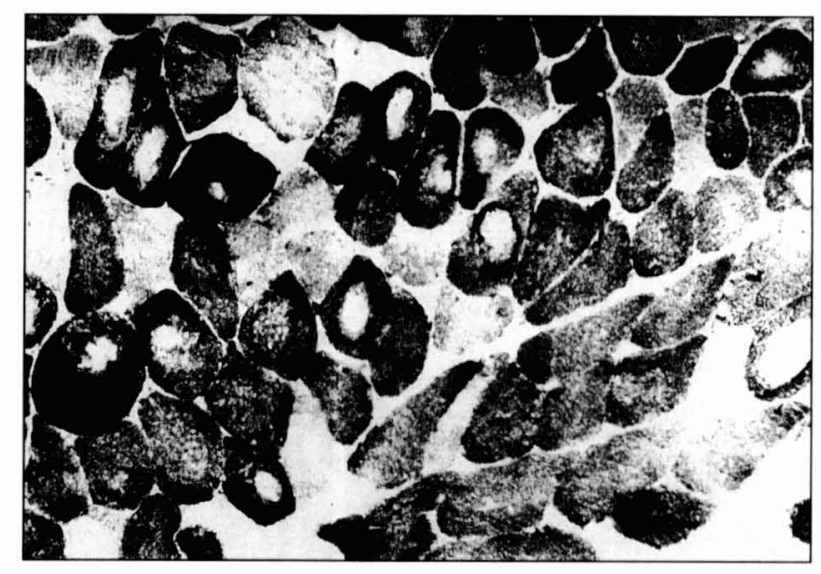

Figura 3. Numerosas fibras musculares muestran una zona clara central rodeada en ocasiones por un halo denso (pseudocores) (DPNH $\times 135$ )

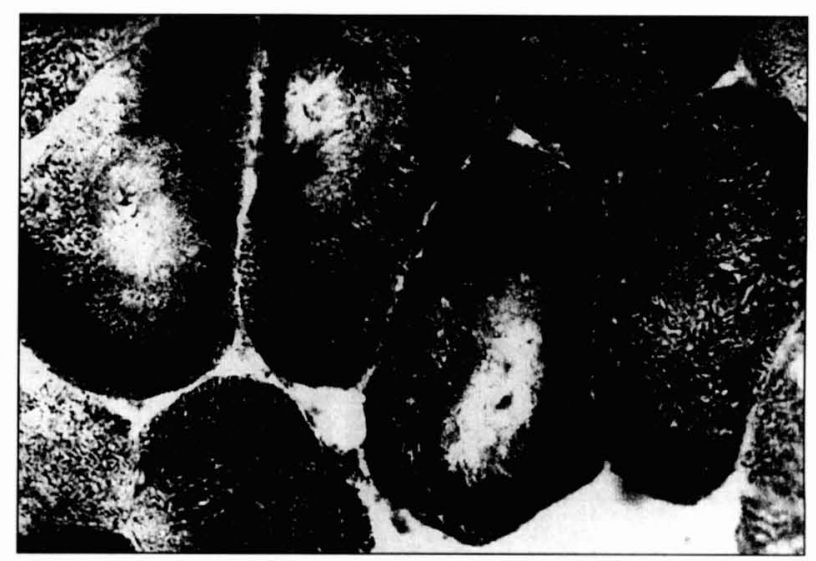

Figura 4. Pseudocores con mayor detalle. La zona pálida está desprovista de mitocondrias y retículo sarcoplásmico. (DPNH x 500). 


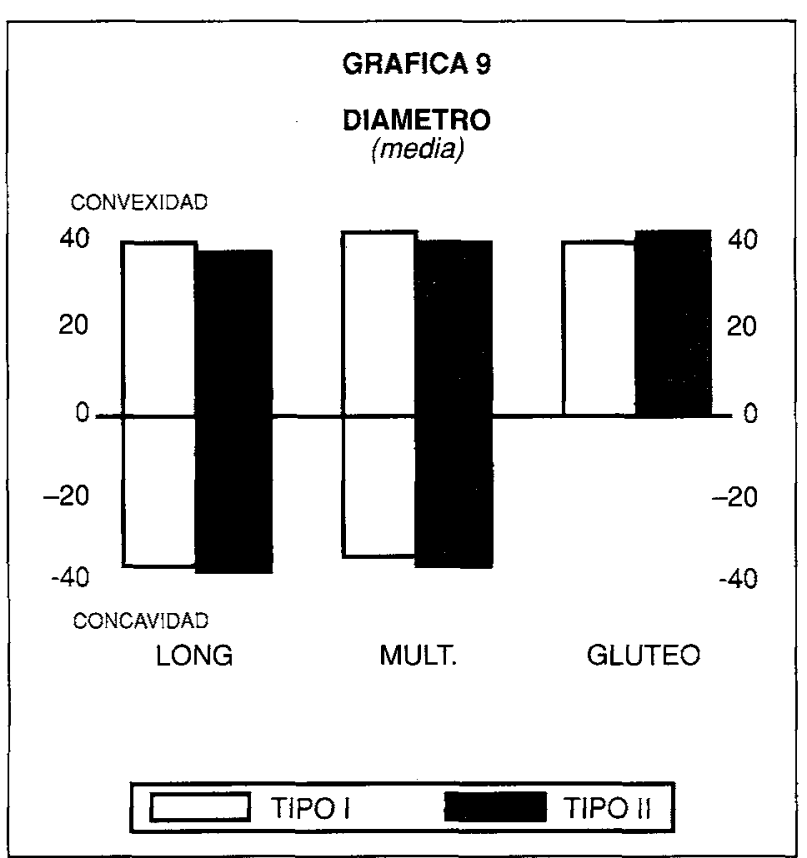

\section{RESULTADOS DEL ESTUDIO MORFOMÉTRICO}

Se realizó una medición en cuanto al número y diámetro de fibras tipo I y II de los músculos paravertebrales longíssimo y multífido a ambos lados de la curva escoliótica y del músculo glúteo del lado derecho correspondiente al lado de la convexidad de la curva y cuyos valores se exponen en la tabla $n^{\circ} 2$.

Se hizo un tratamiento estadístico no paramétrico al no haber controles normales. Se emplearon dos técnicas estadisticas no paramétricas: el test de los signos y el test de Wilconxon.

El test de los signos considera diferencias entre pares de valores de una misma observación asignando signos + al valor mayor y signo - al valor menor. Asimismo asigna una probabilidad del $50 \%$ con una distribución tipo binominial y no considera la magnitud de las diferencias. Por ello y para tener en cuenta la magnitud de las diferencias se utilizó el test de Wilconxon cuyo poder estadístico es más potente que el test de los signos, pues no solamente valora si los datos están a la izquierda o a la derecha de la mediana sino que además a que distancia. Es técnica no paramétrica equivalente a la t de Student para muestras paramétricas.

Con el test de Wilconxon se comparan pares de valores de los distintos tipos de fibras entre ellas y a ambos lados de la curva en cuanto a número y diámetro.

El estudio estadístico del test de Wilconxon se efectuó en ordenador utilizando un paquete estadístico SIGMA.

En resumen se han encontrado valores significativos en los siguientes estudios comparativos (gráficos $9,10,11$ ).

1. Músculo longíssimo (fig. 2):

Número de fibras tipo I mayor que el número de fibras tipo II en la convexidad de la curva.

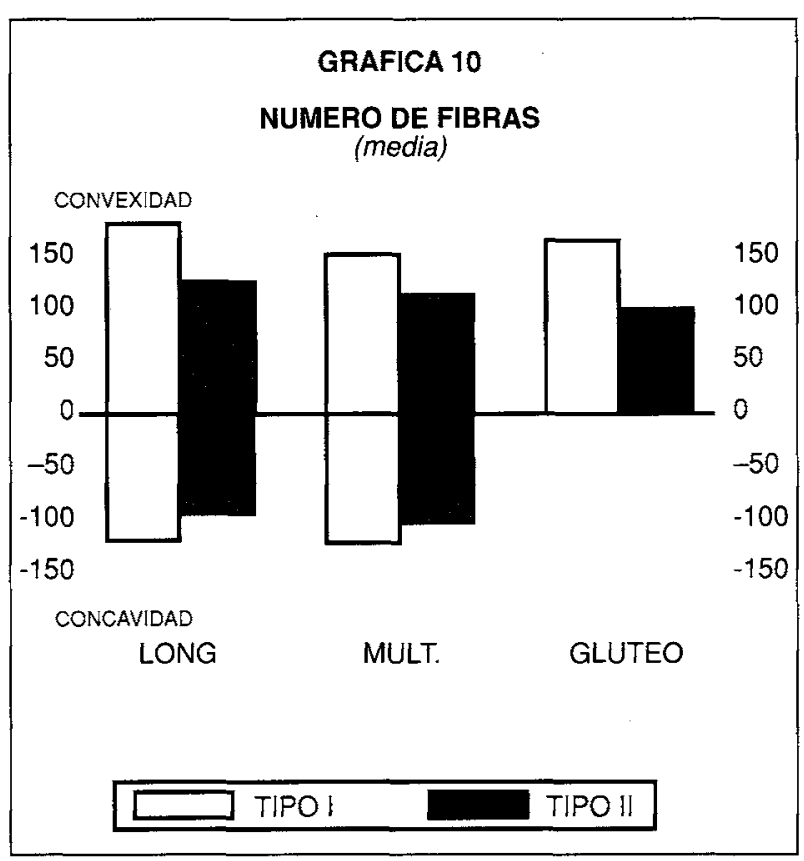

2. Músculo multifíto:

Número de fibras tipo I mayor que el número de fibras tipo II en la convexidad de la curva.

3. Músculo glúteo:

Número de fibras tipo I mayor que el número de fibras tipo II.

4. Músculo longíssimo (fig. 1):

Diámetro de fibras tipo I de la convexidad mayor que el diámetro fibras tipo I de la concavidad.

5. Músculo multífido:

Diámetro de fibras tipo I de la convexidad mayor que el diámetro de fibras tipo I de la concavidad.

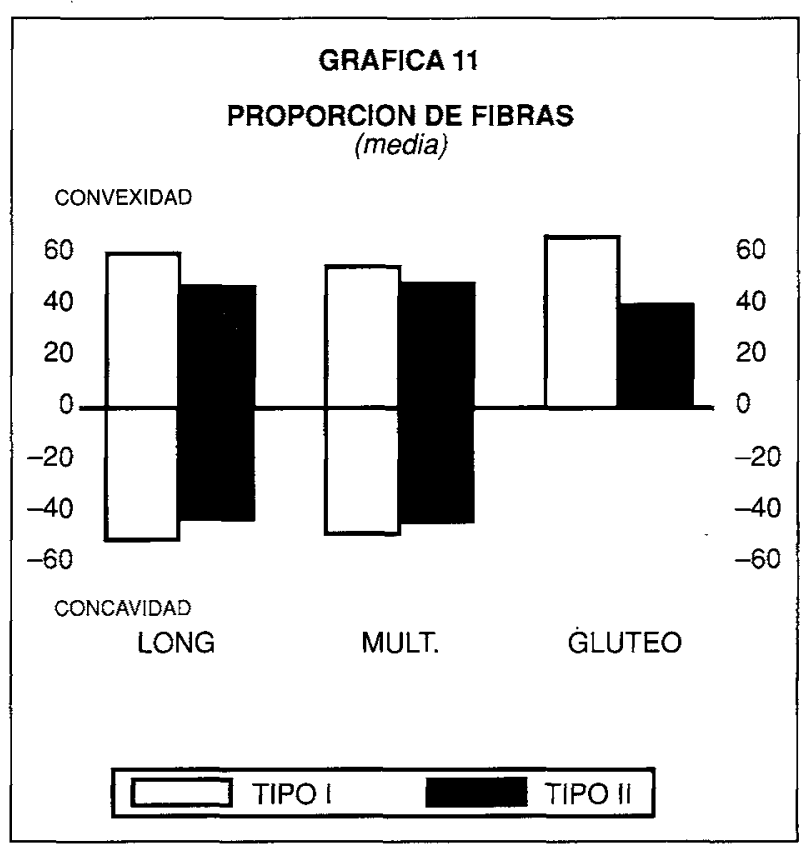


No se han encontrado valores significativos en los siguientes estudios comparativos:

1. Músculo longíssimo:

Número de fibras tipo I en relación al de tipo II en la concavidad de la curva.

Número de fibras tipo I de la convexidad en relación al tipo I de la concavidad de la curva.

Número de fibras tipo II de la convexidad en relación al número de fibras tipo II de la concavidad de la curva.

2. Músculo multífido:

Número de fibras tipo I en relación al de tipo II en la concavidad de la curva.

Número de fibras tipo I de la convexidad en relación al número de fibras tipo I de la concavidad de la curva.

Número de fibras tipo II de la convexidad en relación al número de fibras tipo II de la concavidad de la curva.

\section{Músculo longíssimo:}

Diámetro de fibras tipo I en relación al diámetro de fibras tipo II en la convexidad de la curva.

Diámetro de fibras tipo II de la convexidad en relación al de la concavidad de la curva.

Diámetro de fibras tipo I en relación al diámetro de fibras tipo II en la concavidad de la curva.

4. Músculo multifido:

Diámetro de fibras tipo I en relación al diámetro tipo II en la convexidad de la curva.

Diámetro de fibras tipo II de la convexidad en relación al diámetro de fibras tipo II de la concavidad de la curva.

Diámetro de fibras tipo I en relación al diámetro de fibras tipo II en la concavidad de la curva.

5. Músculo glúteo:

Diámetro de fibras tipo I en relación al diámetro de fibras tipo II.

\section{DISCUSIÓN}

La electromiografía es un buen medio auxiliar a la hora de realizar estudios sobre la acción muscular. Numerosos trabajos han ido aumentando el conocimiento acerca de las funciones de los músculos de la espalda. ${ }^{4,5,54,62,94}$

También se han realizado estudios electromiográficos en sujetos con escoliosis. $1.25,33,38,47,70,71,84,91,92,106,107,08,116,117$

La estabilidad de la columna vertebral está mantenida por músculos. En la columna escoliótica hay un momento de flexión que actúa sobre la columna en el plano frontal, proporcional al grado de escoliosis. Esto significa, que para mantener una postura recta los músculos paravertebrales en el lado convexo tienen que realizar más trabajo que los del lado cóncavo. Esto se ha visto en la mayoría de los enfermos donde una diferencia de amplitud de señal mioeléctrica estaba en relación con un ángulo escoliótico aumentado. La amplitud de señal mioeléctrica aumenta con el aumento de fuerza ejercido. Esto implica que las diferencias de lado son probablemente secundarias a la curvadura de la columna.

Aunque no se pudieron realizar los registros en todas las posiciones a todos los enfermos, ya que algunos sufrieron una lipotimia o no pudieron soportar las molestias de la aguja del electrodo generalmente en la posición de bipedestación se obtuvieron suficientes electromiogramas para entablar una discusión y establecer unas conclusiones.

Se ha utilizado la clasificación de King para diferenciar las curvas torácicas de las curvas lumbares, ya que no se puede englobar a todas las escoliosis de una manera única y los resultados dependerán del tipo de curva y de su magnitud.

En las curvas tipo K1, donde la curva lumbar es de mayor magnitud que la curva torácica, en posiciones de decúbito y reposo a nivel de la curva torácica había actividad mioelectrica en el lado convexo en 2 enfermos en el músculo longíssimo y multífido. En el cóncavo fué nula. En la posición de bipedestación se registró actividad solo en 1 caso en ambos músculos longissimo y multífido. Al igual que en la posición de decúbito no se registró ninguna actividad en el lado cóncavo.

A nivel de la curva lumbar en posición de decúbito se registró actividad en el lado convexo en 1 caso en ambos músculos. Sin embargo en la posición de bipedestación se registró actividad en 3 casos a nivel del músculo longissimo y en los 4 casos en el músculo multífido. En el lado cóncavo en las dos posiciones de decúbito y bipedestación no se registró actividad alguna.

En resumen se ha visto mayor actividad mioeléctrica en este grupo de curvas en el lado convexo de la curva lumbar o curva mayor, en la posición de bipedestación.

En las curvas $\mathrm{K} 2$ donde la curva torácica es mayor que la curva lumbar, en la posición de decúbito y reposo, se observó actividad mioeléctrica en el lado convexo de la curva torácica en ambos grupos musculares longíssimos y multífidos en casi todos los enfermos, siendo la actividad mioeléctirca en el lado cóncavo de 0 en 11 casos y de 1 en dos casos en el músculo longissimo y actividad 1 en tres casos en el músculo multífido. En la posición de bipedestación esta diferencia de actividad en ambos lados y músculos era muy poco significativa.

A nivel de la curva lumbar en la posición de reposo se observó actividad en el lado convexo en 4 enfermos en el músculo longíssimo y en 2 enfermos en el músculo multífido mientras que en bipedestación solo se observó actividad en el lado convexo en un caso a nivel del músculo multífido. En el lado cóncavo no se registro actividad en ningún enfermo en las dos posiciones. 


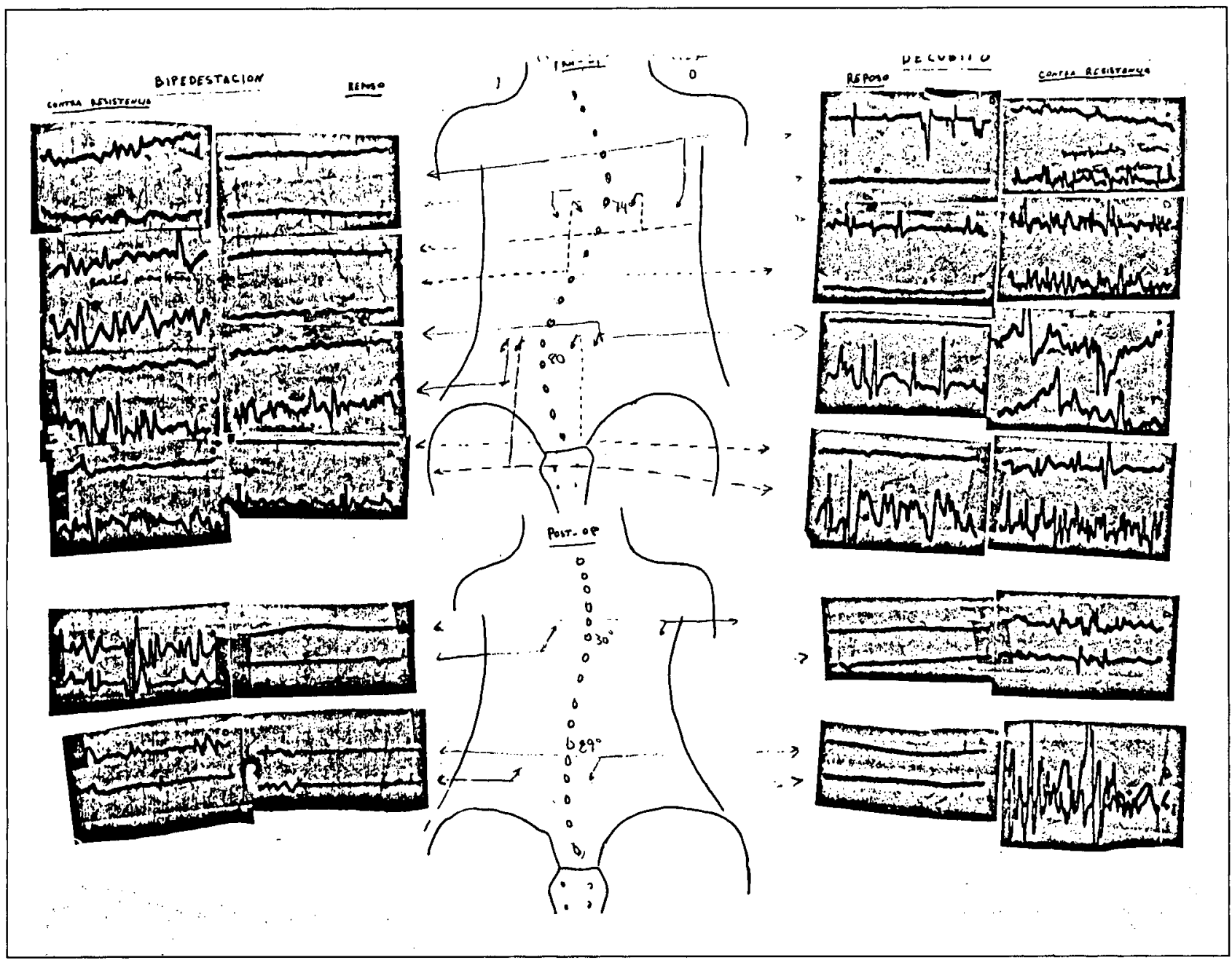

Curva tipo K-I. Actividad electromiográfica en convexidades de ambas curvas, más evidente en la curva lumbar de $80^{\circ}$ mayor que la torácica $\mathbf{7 4}^{\circ}$. En el postoperatorio desaparecen las actividades electromiográficas en ambas curvas.

En este grupo de curvas, por tanto, la mayor actividad mioeléctrica registrada ha sido a nivel del lado convexo de la curva mayor o torácica en la posición de decúbito y en ambos músculos longissimo y multífido.

En un caso de curva $\mathrm{K} 3$, con una curva torácica de $43^{\circ}$ y curva lumbar prácticamente inexistente $15^{\circ}$, sólo existía actividad mioeléctrica en el lado convexo de la curva torácica, en posiciones de decúbito y bipedestación en el músculo longíssimo. Las actividades registradas en la curva lumbar eran nulas a ambos lados y músculos.

En un caso de curva K5 con una curva superior torácica de $84^{\circ}$ y curva inferior toracolumbar de $98^{\circ}$, había actividad mioeléctrica en el lado convexo de la curva superior torácica en el músculo longíssimo en las posiciones de decúbito y bipedestación y multífido en la posición de decúbito. En la curva torácica inferior la actividad fué muy significativa en el lado convexo, a nivel del músculo multífido, en las posiciones de decúbito y bipedestación. En el músculo longissimo se registró actividad en el lado convexo en la posición de decúbito.

En las curvas lumbares a nivel torácico solo se observó actividad en un caso en el musculo longíssimo del lado convexo en la posición de bipedestación y reposo y en el lado cóncavo del músculo longíssimo en la posición de decúbito reposo. A nivel lumbar donde se registró mayor actividad fué en el músculo longíssimo del lado convexo, en la posición de bipedestación y reposo. En el lado cóncavo no se registró ninguna actividad.

Por tanto, en este grupo se registró mayor actividad mioeléctrica en el lado convexo de la curva mayor lumbar en la posición de bipedestación al igual de lo que ocurría en las curvas tipo K2.

Los valores de actividad mioeléctrica en posiciones contra-resistencia en decúbito y bipedestación, eran altos en ambas curvas y en ambos lados convexo y cóncavo, independientemente del tipo curva, aunque eran ligeramente superiores en la posición de decúbito en relación a la postura de bipedestación, esto es debido a que la pelvis y el sacro, sitio de origen de los erectores de raquis, se fijan mejor en decúbito por lo que la fuerza de acción de estos es más potente.

La electromiografía se practicó a los 6 meses de la intervención quirúrgica en 17 enfermos. En los 13 


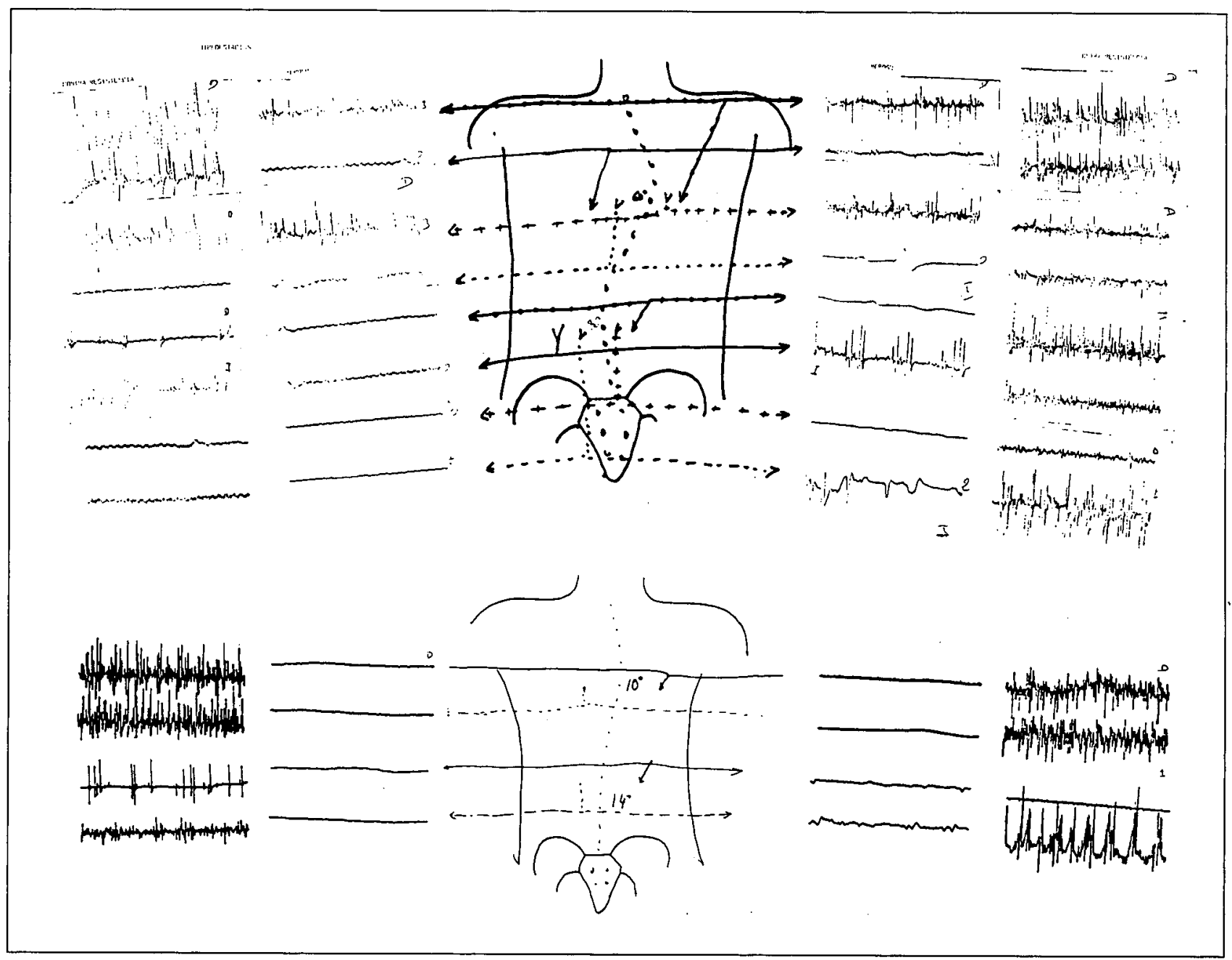

Curva tipo K-II: Aumento de actividad electromiográfica en convexidades de ambas curvas más significativa en la curva torácica de $60^{\circ}$ que en la lumbar de $30^{\circ}$. En el postoperatorio desaparecen las actividades electromiográficas.

enfermos que se practicó vía posterior, no se observaron potenciales de denervación muscular, de donde se deduce que el tiempo de 6 meses es suficiente para renervar a estos músculos en el caso que hubiese habido denervación trás la cirugía. Se observó que en las posiciones de decúbito y bipedestación en la actitud de reposo, la actividad mioeléctrica había desparecido en el lado convexo en la mayoría de los enfermos, es decir, se habían igualado las actividades mioeléctricas.

Lo mismo ocurrió en los 4 de los enfermos con curvas lumbares o toraco-lumbares que habían sido intervenidos por vía anterior, e instrumentadas con técnica de Zielke donde no había habido posibilidad de denervación de los músculos longíssimo y multifido. Las actividades mioeléctricas de ambos lados se hicieron nulas excepto en un caso en la posición de bipedestación en el que se observó actividad mioeléctrica de valor 1 en el longíssimo del lado convexo.

Las actividades mioeléctricas en las posiciones contra resistencia no variaron prácticamente en relación al preoperatorio.
Los resultados de este estudio, por tanto, indican que hay una actividad mioeléctrica aumentada en el lado convexo de la curva, estando de acuerdo con los estudios realizados por Lefebvre y cols. ${ }^{47}$, Alexander y Season ${ }^{2}$, Zuck $^{117}$, Donovan y cols. ${ }^{25}$ Redford y cols. ${ }^{70}$ y Schultz y cols. ${ }^{76}$. La intepretación de esta mayor actividad mioeléctrica en el lado convexo de la curva escoliótica, se debe al mayor sobreesfuerzo que realizan los músculos del lado convexo y a la menor actividad de los músculos del lado cóncavo.

Estos resultados se contradicen con los estudios realizados por Zetterberg y cols. ${ }^{116}$ que señalan como anómalo el lado cóncavo de la curva escoliótica, donde no existe actividad mioeléctrica; ya que a los 6 meses de la intervención quirúrgica, se ha observado actividad mioeléctrica nula en ambos lados de la curva escoliótica (convexo y cóncavo), tanto en enfermos que habían sido intervenidos por vía posterior donde podría existir un factor de denervación de la musculatura paravertebral por la cirugía hecho que no ocurre como demostraron Donvoan y cols. ${ }^{25}$, como en los intervenidos por vía anterior, donde no existe denervación en estos músculos debido a la cirugía. 
Por tanto el hecho de corregir y estabilizar las curvas, hace que no haya sobrecarga de la musculatura paravertebral en el lado convexo en relación al lado cóncavo y como consecuencia de ello desaparece la actividad mioeléctrica que existía en este lado convexo.

En el estudio de la musculatura paravertebral, realizada a microscopio óptico, se observó, en los 15 enfermos analizados, lo siguiente:

- Aumento del tejido conectivo endomisial en 3 enfermos.

- Fibras atróficas con las características de la atrofia por denervación en el músculo longíssimo de la concavidad de la curva en dos enfermos.

- Fibras con abundante glucógeno y lípidos en los músculos en siete enfermos.

- Zonas de ausencia de tinción con las enzimas oxidativas generalmente grandes y únicas, de aspecto muy parecido a los cores, afectando a las fibras tipo I de los músculos multífido en la convexidad a seis enfermos, en la concavidad a cuatro y en el longíssimo de la concavidad a uno.

Estos hallazgos indicarían la existencia de leves cambios miopáticos o lesiones asociadas a denervación.

En el estudio morfométrico de los músculos longíssimo y multífido se encontró: a) un predominio de fibras tipo I sobre fibras tipo II en ambos lados de la curva y b) un aumento significativo de fibras tipo I sobre fibras tipo II en ambos músculos longíssimo y multífido en el lado convexo; hallazgos similares a los observados por Yaron y cols. ${ }^{109,100,111,112,113}$, Sahgal y cols. ${ }^{72}$, y Bylund y cols. ${ }^{17}$

En un enfermo se observó un predominio de fibras tipo II en el músculo longíssimo a ambos lados de la curva y en otro enfermos este mismo predominio de fibras tipo II pero en el músculo multífido. No se sabe interpretar el porqué de este predominio de fibras tipo II en estos dos enfermos ya que la magnitud de la curva y edad eran similares al resto.

En los hallazgos observados por Sahgal y cols. ${ }^{72}$ también describen un caso con predominio de fibras tipo II en el músculo longíssimo, en un estudio realizado en catorce enfermos con escoliosis idiopática sin explicar el proqué de este hallazgo.

En el músculo glúteo mayor derecho, coincidiendo con la convexidad de la curva torácica, se observó un predominio claro de fibras tipo I sobre tipo II en todos los enfermos.

En cuanto al diámetro de las fibras musculares se vió una disminución significativa de éste en las fibras tipo I en los músculos longíssimo y multífido de la concavidad de la curva, en relación a las fibras tipo I de ambos músculos en el lado convexo, por lo cual cabría pensar que hay una atrofia en las fibras tipo I del lado de la concavidad corroborando, por tanto, los hallazgos de Yarom y cols. y no estando de acuerdo con los de Sahgal y cols. ${ }^{72}$ que concluyen diciendo que existe una atrofia de fibras tipo II en la concavidad de la curva escoliótica.
El porcentaje mayor de fibras tipo I sobre las fibras tipo II en el lado convexo parece que podría ser una respuesta fisiológica de la musculatura paravertebral, debido a que estos músculos del lado convexo tratan de equilibrar la columna vertebral. Se produce, por tanto, como respuesta fisiológica a la sobrecarga que tiene el lado convexo, un cambio de fibras tipo II fásicas o de contracción rápida, a fibras tipo I tónicas de contracción lenta, más resistentes a la fatiga y con alta capacidad oxidativa.

La atrofia de fibras tipo I de la musculatura paravertebral del lado cóncavo de la curva escoliótica sería secundaria a la mayor sobrecarga que existe en este lado.

Correlacionando estos hallazgos histopatológicos con los estudios electromiográficos realizados en estos enfermos escolióticos se obseva:

Que en la musculatura paravertebral del lado convexo hay mayor actividad mioeléctrica que en el lado cóncavo, donde es prácticamente nula. Lo que significa que la mayor demanda de trabajo muscular, como es el lado convexo de la curva escoliótica, requiere mayor número de fibras tipo I, y en consecuencia, registra mayor actividad electromiográfica; mientras que, a menor demanda de trabajo se produce menor número y mayor atrofia de fibras tipo I y menor actividad electromiográfica.

Realizados registros electromiográficos a los seis meses de la intervención de estos enfermos, una vez corregidas y estabilizadas sus curvas, se observa que las actividades mioeléctricas de los músculos paravertebrales son prácticamente nulas a ambos lados de la curva. Estos resultados se dieron tanto utilizando la intervención por vía posterior, donde ya no existe denervación de la musculatura paravertebral, como demostraron Donovan y cols. ${ }^{25}$, ó trás la intervención por vía anterior, donde no existe alteración de la intervención de la musculatura por la acción de la cirugía.

Esto hace pensar que no existe una disfunción neuromuscular como causa etiopatogénica única de la escoliosis idiopática.

Por lo tanto los resultados de estos estudios están en desacuerdo con las teorías de Yaron y cols. 109,110,111,112,113, Sahgal y cols. ${ }^{72}$, Jean Chiu y cols. ${ }^{41}$, Bylund y cols. ${ }^{17}$ que opinan que la escoliosis idiopática es causada por una miopatía de carácter leve. En cambio son coincidentes con Zetterberg y cols. ${ }^{115}$, Ford y cols. ${ }^{28} \mathrm{Hsu}$ y cols. ${ }^{39}$ y Gibson y cols. ${ }^{30}$, que opinan que hay una adaptación secundaria de las fibras musculares a la curva escoliótica.

Sin embargo, no se puede excluir el que exista una desviación patológica de fibras en la musculatura paravertebral y que fuese un factor primario en la etiopatogenía de la escoliosis idiopática. Una razón de esto es que la mayoría de las curvas han sido siempre convexas hacia el lado derecho. Podría ser que en ciertos individuos, sus músculos paravertebrales en el lado izquierdo tuvieran menos fibras tipo I y en consecuencia ser menos resistentes a la fatiga que los del lado derecho. El resultado de este desequilibrio muscular hace que se produzca la curva escoliótica. 
Esto podría ser más evidente en la etapa pre-puberal momento en que se produce un crecimiento rápido. $\mathrm{Al}$ coincidir con una disminución de la cifosis torácica fisiológica, hace que el centro de rotación de la vértebra torácica se acerque al centro de rotación del raquis.

Este acercamiento del eje de rotación de la vértebra torácica al eje de rotación del raquis, facilita que la vértebra rote hacia el lado donde los rotadores son más potentes, es decir, hacia el lado derecho o convexo, de tal forma que el arco vertebral rota hacia el lado izquierdo o cóncavo y el cuerpo hacia el lado derecho o convexo. Si se sobrepasa un límite de rotación, la musculatura a ambos lados de los arcos posteriores unifica sus acciones en un mismo sentido, propiciando que se dispare la progresión de la curva escoliótica.

Después de haber hecho una revisión bibliográfica de todas las posibles causas etiopatogénicas (genéticas $19,20,100,101$, elementos estructurados de la columna vertebral ${ }^{44,63,114}$, estructura del colágeno ${ }^{15,58,93}$, sistema endocrino $53,79,87,88$, factores biomecánicos ${ }^{23}$, sistema del equilibrio $^{36,73,74,87,99,102,103,104}$ factores metabólicos ${ }^{67}$, escoliosis experimental ${ }^{8,22,34,42,45,46,48,56,65,66,78,83}$ y de los estudios musculares desde el punto de vista electromiográfico e histoquímico y morfométrico se puede concluir, de forma general que la enfermedad que se está buscando es de causa multifactorial, como la luxación congénica de cadera, pie zambo, mielomeningocele y paladar hendido.

La investigación no está agotada en su totalidad. Se debe seguir investigando sobre esta deformidad de la columna y realizar un estudio multidisciplinario. Por tanto, colaborar con el bioquímico, neurofisiólogo, ingeniero biomecánico, neuropatólogo, endocrino etc.

\section{CONCLUSIONES DEL ESTUDIO ELECTROMIOGRÁFICO}

$1^{a}$. Se deduce que la mayor actividad electromiográfica en el lado convexo de la curva escoliótica, está en relación al mayor esfuerzo que realizan los músculos de este lado, intentando equilibrar la columna vertebral y que la ausencia de ésta actividad en el lado cóncavo está en relación con la no actividad muscular.

2 ${ }^{\text {a }}$ Se constata que los músculos longíssimo y multífido contribuyen con su actividad a la aparición de potenciales eléctricos aumentados en ambos lados de la curva escoliótica, en actitudes de esfuerzo contra resistencia, en las posiciones de reposo y bipedestación.

$3^{\text {a }}$ La ausencia de actividad electromiográfica en el músculo longíssimo a ambos lados de la curva esoliótica (una vez corregida y estabilizada), se debe a que no existe lesión muscular como causa etiopatogénica única de la escoliosis idiopática.

4․ A diferencia de los resultados obtenidos en la posición de reposo se constata, que no hay variación de las actividades mioeléctricas, en las mediciones realizadas en el músculo longíssimo a los seis meses del postoperatorio, en las actitudes de esfuerzo contra resistencia, en posiciones de decúbito y bipedestación.

\section{CONCLUSIONES DEL ESTUDIO HISTOQUÍMICO Y MORFOMÉTRICO}

$1^{\text {a }}$. Se observa la existencia de leves cambios miopáticos o lesiones asociadas a denervaciones en músculos de ambos lados de la curva escoliótica.

$2^{\text {a }}$. Existe un aumento significativo del número de fibras tipo I sobre las de tipo II en el músculo longíssimo y multífido en la convexidad de la curva escoliótica.

$3^{\text {a }}$. Se observa un aumento significativo del número de fibras tipo I sobre las de tipo II en el músculo glúteo del lado convexo de la curva.

4․․ Hay un aumento significativo del diámetro de las fibras tipo I de la convexidad sobre el diámetro de fibras tipo I de la concavidad en los músculos longíssimo y multífido.

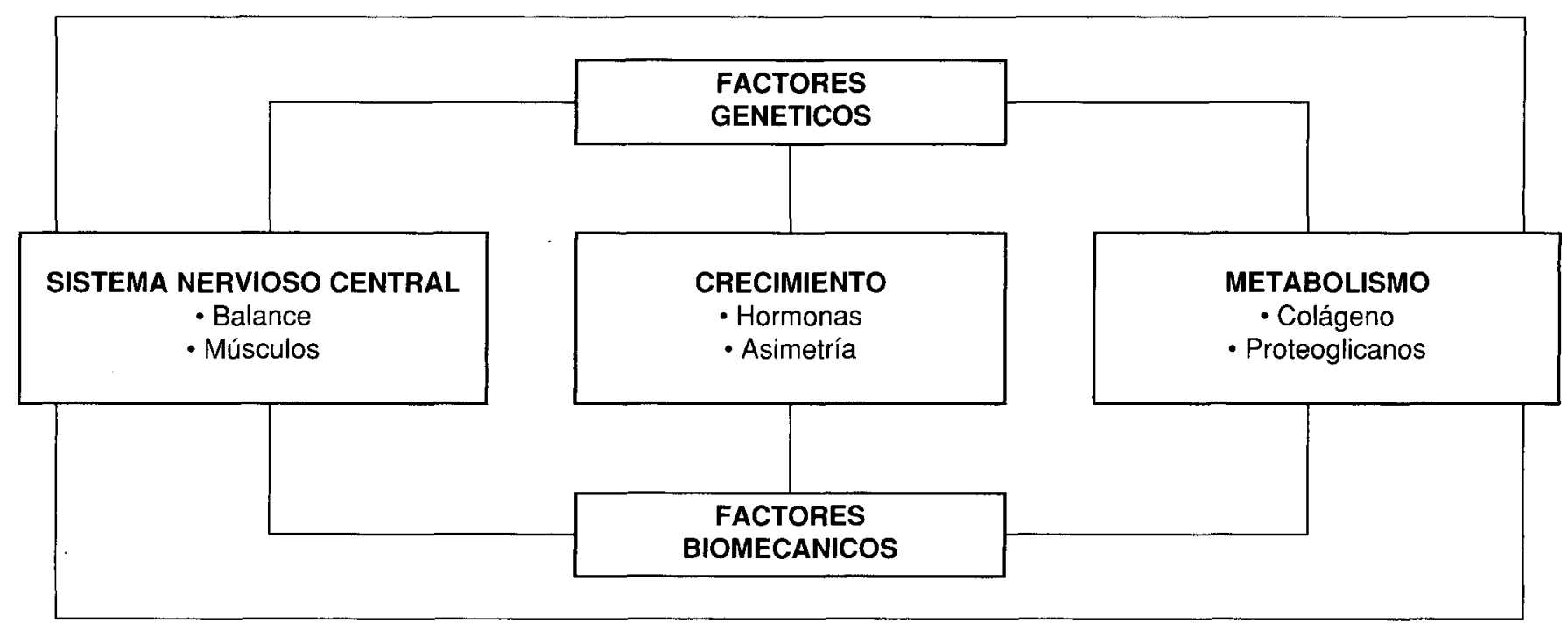


5 . Existe una correlación entre mayor número y diámetro de las fibras tipo I con aumento de la actividad electromiográfica en la convexidad de la curva escoliótica.

\section{AGRADECIMIENTO}

Deseo expresar mi agradecimiento a la Prof. Dra. Ana Cabello jefe de sección del Servicio de Anatomía Patológica del Hospital 12 de Octubre y al Dr. Félix del Campo médico adjunto del Servicio de Neurofisiología del Hospital de Getafe por la ayuda recibida en la realización de esta tesis doctoral.

\section{BIBLIOGRAFÍA}

1. Alexander, M.A., Bunch, W., and Ebberson, S.: Can experimental dorsal rhizotomy produce scoliosis. J. Bone Joint Surg. 54 A 1509 , 1972.

2. Alexander, A A Season, Eh. Idiopathic scoliosis. An electromiographic study. Arch. Phys. Med. Rehabil. 59, 314, 1978.

3. Alexeeva, A.A., Chemukin, A.A.: Disorder of the neuromuscular balance one the factors of development of scoliosis (Experimental study). Ortop.- Traumatolo-Protez, 12, 24, 1978

4. Allen, D.E.L: Muscle action potencials used in the study of dynamic anatomy. Brit. J. Phys. Med. 11, 66, 1948

5. Andrssen, G.B., Murphy, R.W., Ortengren, R., Nachemson, A.L., Effstron, G., bronson, H.: The sitting pposture. An electromyographic and discometric study. Orthop. Clin. North Am. (1), 105, 1075.

6. Anderssen, G.B., Murphy, R.W., Ortengren, R., Nachemson, A.L., Effstron, G., Bronson, H.: The influence of backrest inclination and lumbar support on lumbar lordosis. Spine 4, 52, 1979.

7. Archer, I.A., Dickson R.A.: Spinal Deformities basic principles. Current Orthopaedics 3, 72, 1989.

8. Barrios, C. Tuñon, MT., De Salis, I., Beguiristain, JL. and Cañadell, J.: Scoliosis Induced by medullary damage. An experimental study in rabbits. Spine 12, 5, 433, 1987

9. Basmajian, J.V.: Muscle Alive. The back. The Williams and Wilkins Company. Baltimore, 281-283, 1967

10. Beguiristain, JL.: Escoliosis experimental en ratas bípedas. Rev. Orotp. Traum. 18, 367, 1974.

11. Beguiristain, JL., De Salis, J., Ornifo, A., and Cañadell, J.: Experimental scoliosis by epihysiodesis in pigs. Int. Orthop. 3, 317, 1980.

12. Belenky, VE.: The mechanism of formation of the spine deformity in scoliosis. Excerpta Medica, Orthopedic Surg, 23, 4, 1978.

13. Bisgard, JD.: Experimental thoracogenesis scoliosis. J. Thoracic Surg. 4, 435, 1935 .

14. Bohmer, D., Norte, B.: Biochemical factors in the etiology of scoliosis. Z. Orthop. 110/2, 137,1972.

15. Bradford, DS., Oegema, TR., and Braun, DM.: Studies on skin fibroblast of patients with idiopatic scoliosis. Clin. Orthop. 126: III, 1977

16. Bushell, GR., Ghosh, P., Taylor, TKF.: Collagen defect in idiopathic scoliosis. Lancet 2, 94, 1978

17. Bylund, P., Jansson, E., Dahlberg, E and Erikson, E.; Muscle Fiber Types in thoracic erector spinae muscles. Clin. Orthop. 214, 222, 1987.

18. Cañadell, J., Beguiristain, JL., González Iturri, J., Reparaz, B. and Gili, JR.; Some aspects of experimental scoliosis. Arch. Orthop. 'Traumat. Surg. 93, 75, 1978.

19. Cotrel, MH.: Le facteur genetique dans la scoliose idopatique. These. Paris, 1974

20. De George, F.V. and Fischer, RL.: Idiopathic scoliosis. Genetic and enviromental aspects. J. Med. Genet. 4, 251, 1967

21. De Salis, JA.: Escoliosis experimental por lesión vascular. M.D. Thesis Universidad de Navarra. Pamplona, 1977.

22. De Salis, JA., Beguiristain, JL. and Cañadell J.: The production of experimental scoliosis by selectiva arterial ablation. Int. Orthop. 3 , $311 ; 1980$.

23. Dickson, RA., Lawton, JO., Archer IA, Butt, WP.: The pathogenesis of idiopathic scoliosis: biplanar spinar asymmetry. J. Bone Joint Surg. (br) 66B, 8, 1984

24. Donish, EW. and Basmajian, JV.: Electromiography of deep back muscles in man. Am. J. Anat., 133, 25, 1972.

25. Donovan, WH., Dwywer, AP., Bedbrook, GM.: Electromyographic activity in paraspinal musculature in patients with idiopathic scoliosis before and after Harrington instrumentation. Arch Phys Med.
Rehabil. 61/9, 413, 1980.

26. Fidler, MW. and Jowett RL.: Muscle imbalance in the etiology of scoliosis.. J. Bone Joint Surg., 58 B, 1, 1976.

27. Fillio, NA., Thompson, MW.: Genetic studies in scoliosis. Proceedings of the Scoliosis Research Society. J. Bone Joint Surg. 53 A, 1991, 1971.

28. Ford, DM., Bagnall KM., Clements CA. and McFadden KD Muscles spindles in the papraspinal musculature of patients with adolescent idiopathic scoliosis. Spine, 13, 461, 1988

29. Ford, DM., Bagnall, KM., McFadden, KD.; Paraspinal muscle imbalance in adolescent idopathic scoliosis. Spine, 9/4, 373, 1984.

30. Gibson, JNA., McMaster, MJ., Scrimgeour, CM., Stoward, PJ., Rennie, MJ.: Rates of muscle protein synthesis in paraspinal muscies Lateral disparity in children with idiopahic scoliosis. Clin. Sci. $75.1,79-83$.

31. Green, RJL.: Histochemistry and ultrastructure oif the paraspinal muscles in idiopathic scoliosis and in control subjects. Med. Lab. Sci. 38/3, 197, 1981.

32. Gregoric, M., Pecak, F., Trontelj, IV., Dimitrijevic, MR.; Postural control in scoliosos. A statokinesimetric study in patients with scoliosis due to neuromuscular disorders and in patients with idiopathic scoliosis. Acta Orthop. Scand., 52, 59, 1981

33. Gueth, V., Abbink, F.: Electromyographical and kinesiological investigations: Comparison of congenital and idiopathic scoliosis. Z. Orthop. Thre. Genzgeb, 118-2, 165, 1980.

34. Gurpide, C.: Comportamiento de la médula en la escoliosis experimental. Tesis doctoral, Universidad de Navarra, España, 1977.;

35. Haderspeck, K., Schultz, AB.: Progression of idiopathic scoliosis. An analysis of muscle actions and body weitht influences. Spine 6 , $447,1981$.

36. Herman, R.: Postural and ocular motor control in patients with idiopathic scoliosis. Pathogenesis of idiopathic scoliosis. Ed. Scoliosis Reseearch Society, 193, 1984.

37. Hoogmastens, MJ., Basmajian, JV.: Postural tone in the deep spinal muscles of idiopathic scolisosis patients and their siblings. Electroendefalograph Clin. Neurophisiol 16, 93-114, 1976.

38. Hoogmanstens, MJ., Stuyck, J.: First results with vibration elecktromyography as a differential measurement of the postural tone in the left and right spinal muscles. Agressologie 18, 34I-343, 1978.

39. Hsu, JD., Slager, UT., Swand, SM., Robinson, MH.: Idiopathic scoliosis: A clinical, morphometrc and histopathological correlation. J. Pediatr. Orthop., 155, 147, 1988.

40. Hunter, KRE., Bradford, DS., oegema TR: Biochemistry of the intervertebral disc in scoliosis. Presented at the Annual Meeting of the Orthopaedic Research Society, Dallas, Texas, 135, 1978.

41. Jean-Cheng Chiu, Schun-ichi Inove, Toshio Naguna: Morphological studies on muscle fibers and neural elements of the erection spinae muscle in 60 consecutive scoliotic patient. 23rd. Annual Meeting Scoliosis Research Society, Baltimore, Maryland, 1988.

42. Kawate, S.: Experimental scolisois produced lby stereotaxic destruction of posterior part of the hypothalamus in bipedal rats. Shakoku Act Med. 32, 195, 1976.

43. Kazmin, AJ., Fixzczanko, WJ.: On the importance of the nucleus pulposus in scoliosis formation. Chir narzadow Ruchu Ortop. Pol. $35,493,1970$.

44. Knutson, F.: Vertebral genesis of idiopathic scoliosis in children. Acta Radiol. 4, 395, 1966.

45. Langenskold, A., and Michelsson, JE.: Experimental progressive scoliosis in the rabbit. J. Bone Joint Surg. 43A, 115, 1961

46. Lawton, JO. and Dickson, R.: The experimental basis of idopathic scoliosis. Clin. Orthop. 210, 9, 1986.

47. LeFebre, J., Triboulet, Chassevant, A and missirley, MF.; electromyographic data in idiopathic scolisos. Arch. Phys. Med. 42, 7120, 1961.

48. Liszka, O.: Spinal cord mechanisms leading to scoliosis. Acta Med. Pol. $2,45,1961$

49. Low, WD., Chew ED., Kung, LS., Hsu, LCS., Leong, JCY.: Ultrastructure of nerve fibers and muscle spindles in adolescent idiopathic scoliosis. Clin. Orthop. 174, 217, 1983.

50. Lloyd Roberts GC., Pincott, JR., MC Meniman, P., Bayley, IJL., Kendall, B.: Progressionin idiopahic scoliosis. J. Bone Joint Surg. (Br.) $60,451,1978$.

51. MacEwen, GD.: Experimental scoliosis In Zorab, P..A. (od). Proceedings of a second symposium on scoliosis. Causation. Edimburgh, E.S. Livingstone, 18, 1968.

52. macEwen, GD.: Experimental scoliosis. Clin. Orhtop. 93, 69, 1ç973.

53. Misol, S., Ponseti, IV., Samaan, N., Bradbury, JT.: Growthk hormone blood levels in patients with idiopathic scoliosis. Clin. Orthop. $81,122,1971$.

54. Morris, IM., Benner, G and Lucas: An electromyographic study of the instrinsic muscles of the back in man. J. Anat. 96, 509, 1962.

55. machemson, A., Shalstrand, T.: Etiologic factors in adolescent idio- 
pathic scolisis. Spine, 176, 1977.

56. Nakagawa, Y.: An experimental study of postural reflex in scoliosis. Shikoku Acta Med. 32, 155, 1976.

57. Nieto, JM., Martínez, AC.: Electromiografía de la bipedestación de la marcha normal. Rehabilitación, 11, 51, 1981.

58. Nordwall, A.: Studies in idiopathic scoliosis.. Acta Orthop. Scand. 150 suppl. 9, 1973.

59. Ovalle, W., Tredwell, S.: The multifidus myotendon junction: a morphological marker for idiopathic scoliosis. Pathogenesis of idiopathic scoliosis. Ed. Scoliosis Research Society 169, 1984.

60. Parrilla, P., Perricone, G.: Electromygraphy in the idiopathic scoliosis. Medesp., 71, 153, 1974

61. Paul of Aegine. Collected works. Translated for sydenhan Society by F. Adams. London $1834 \mathrm{et}$.

62. Pauly, JE.: An electromyographic analisis of certain movements and exercises. Anat. Rec. 155, 223, 1966.

63. Pedrini, Va., Ponseti, IV. and Dohrman, SC.: Glucosaminoglycans of inrervertebral disc in idiopathic scolisois. J. Lakb Clin. Med. 82, 938, 1973.

64. Pincott, JR.: Observations on the afferent nervous system in idiopathic scoliosis. In Scoliosis 1979, Edited by PA Zorab and D. Siegler, London Academic Press, 1980.

65. Pincott, JR, and Taffs, LF.: Experimental scoliosis in primates. A neurological cause. J. Bone Joint Surg. 64B, 503, 1982.

67. Ponseti, JV., Baird.: Scoliosis and dissecting aneurysm of the aorta in rats fed with lathyrus odoratus seeds. Am. J. path., 82, 1059, 1952.

68. Ponseti, IV., Pedrini, V., Wynne-Davies, R., Duval-Beaupere, G.: Pathogenesis of scoliosis. J. Clin. Orthop. 120, 268, 1976.

69. Rappapart, R.: Plasma androgens and LH in scoliotic patients eith premature pubarche. J. Endocrinal Metab. 38, 401, 1974.

70. Redford, JB., Butterworth, TR., Clements, EL. JR.: use of electromyography as a prognostic aid in the management of idopathic scoliosis. Arch Phys. Med. Rehab. 50, 43, 1969.

71. Reuber, M., Mc Neid, Schultz, A., Spencer, D.: Trunk muscle myoelectric activities in idiopathic scoliosis. Spine (in press.)

72. Sahgal, P., Shah, A., Flannagan, N., Schaffer, M., Kane, W., Subramani, W. and Singh, H.; Morphologic and morphometric studies of muscle in idiopathic scoliosis. Acta orpthop. Scand 54, 242, 1983.

73. Sahlstrand, T.; Equilibrium factors in adolescent idiopathic scoliosis: a clinical study including stabilometmry, electroencepahlography and electronystangnography. Goteborg, 1977.

74. Sahlstrand, T., Ortengren, R., Nachenson, A.: Postural equilibrium in adolescent idiopathic. Acta Orthop. Encand. 49, 354, 1978.

75. Saito, A.: The histological and electromyographical studies on back muscles in scoliosis. J. Chiba Medical Sco. 40, 13, 1964.

76. Schultz, A., Arbor, A.: Biomechanical studies of possible causes for the progression of idiopathic scoliosis. Pathogenesis of idiopathic scoliosis. Ed. Scoliosis Research Society 181, 1984.

77. Schwarztzmann, JR. and Miles, M.: Experimental production of scoliosis in rats and mice. J Bone Joint Surg. 27, 59, 1945.

78. Schwartamann, JR. and Miles, M.: Exerimental production of scoliosis in rats and mice.. J. Bone Joint Surg., 34B, 421, 1952

79. Skogland, LB., Miller, JAA., Skotter, A., Fryklund, L.: Serum somatomedin A and non-dialyzable urinary hydroxypproline in girls with idiopathic scoliosis. Acta Orthopp. Scand. 52, 307, 1981

80. Slager, VT. and hsu, JD.; morphomektry and pathology of the pparaspinous muscles in idiopathic scoliosis. Dev - Med- Child Neurol, 28, 794, 1956.

82. Spencer, GSC.: Plasma and muscle sometomedin in scoliotic children scoliosis. Proceedings of a Fifth Sympposium, London, September, 1976

83. Suk, SI., Song, HS., Lee, CK.: Scoliosis induced by anterior and posterior rhizotomy. Spine 14,692, 1989.

84. Takemitsu, Harada, Atsuta, Imani: Asymmetry of trunk muscle endurance inconvex and concave sides of children with idiopathic scoliosis comparing with normal children. 19th. Annual Meeting Scoliosos Research Soceity-182, 1984.

85. Tamura, T.: An experimental study on scoliosis in bipedal rats with brain stem lesion. J. Jpa. Orthop. Ass. 48, 137, 1974.

86. Taylor, TKF., Ghosh, P. and Bushell, GR.; The contribution of the intervertebral disk to the scoliotid deformity. Clin orthop. 156,79 , 1981.

87. Tezuko, A.: Development of scoliosis in cases with congenital organic abnormalities of the brain stem. Tojikushime J. Exp. Med. 18, 49,1971 .

88. Torbjörn, AHL., Kerstin, AW., Ragrar, K.: Twenty four hours growth hormone. Profiles in puberal girls with idiopathic scoliosis. Spine 139, 1988.

89. Tredwell, SJ.: A review of possible neuromuscular factors. Pathogenesis of idiopathic scoliosis. Ed. Scoliosis Research Society
203,1984

90. Tresserra, J.: Escoliosis experimental. Rev. Ortop. Traum. 31, 739, 1969.

91. Tronteli, JV., Fernandez, JM.: Single fiber EMC. in juvenile idiopathic scoliosis. Muscle-Nerv. 11/4, 297, 1988.

92. Trontelj, JV., Pecak, F and Dimitvijovic, MR.: Segmental neurophysiological mechanisms in scoliosis, J. Bone Joint Surg., 61B, 310, 1979.

93. Ven, G., Mehta, MH. and Makson RM.: Characteristicks of collagen from normal and scoliotic human spinal ligament. Biochem Biophys. Acta 757, 259, 1983.

94. Waters, RL. and Morris, JM.: Effect of spinal sup port on the electrical activity of muscles of the trunk. J. Bone Joint Surg. 52A, 51, 1970.

95. Whalen, RG., Ecob, MS.: Two-dimensional electrophoretic analysis of muscle contractile proteins in paptients with idiopathic scoliosis Clin. Chem. (Winston-Salem, NC.), 28/411, 1036, 1982.

96. Willner, S.: Study of growth in girls with adolescent idiopathic structural scoliosis. Clin. Orthoip. 101, 129, 1974.

97. Willner, S.: A study of heigth, weight and menarche in girls with idiopathic structural scoliosis. Acta Orthop. Scand. 46, 71, 1975.

98. Willner, S., Nilsson, KO., Kastrup, K., Bergstrand, CG.: Growth hormone and somatomedin A in girls with adolescent idiopathic scoliosis. Acta Paediatr. Scand. 65, 547, 1976.

99. Wyatt, MP., Barrack, RL., Mubarak, SJ., Whitecloud, TS., Burke, SW.; Vibratory response in idiopathic scoliosis. J. Bone Joint Surg. 68B, 714, 1986 .

100. Wynne-Davies, R.: Familial idiopahic scoliosis. A familial survery. J. Bone Soint Surg. 50B, 24, 1968,.

101. Wynne-Davies, R.: idiopathic scoliosis. Heritable disorders in orthopaedic practice. London, Blackwell Scientific Publication, 166 1973.

102. Yamada, K., Ikata, T., Yamamoto, H., Nakagowas, Y., Tamaka, H. and Tezuka, A. Equilibrium funciton in scoliosis and active correction plaster jacket for the treatment. Tokushima J. Exp. Med. 15 I 1969.

103. Yamada, K, Yamamoto, H. Nikagawa, Y. Tezuka A. Tamura, T. and Kawata, S.: Etiology of idiopathic scoliosis. Clin. Orthop. 184 $50,1984$.

104 yamada, K, Yamamoto, H., Tamura, T, Tezuka, A ; Development of scoliosis under neurological basis particulary in relation with brain stem abnormalities. J Bone Joint Surg. 56A, 1764, 1974.

105. Yamamoto, H.: A postural desequilibrium as an etiological factor in idiopathic scoliosis. 17th. Annual Scoliosis Research Society, Denver, 1982

106. Yamamoto, H.: A postural desequilibrium as an etiological factor in idiopathic scoliosis. Pathogeneisi of idiopathic scoliosis. Ed. Scoliosis Research Scoety 211, 1984.

107. Yamamoto, H., Tani, T., Sadahiro, T., Kawauchi, T., Nakatani, K. A neurophysiological pronostication in idiopathic scoliosis. Presented at the Annual Meeting of the Scoliosis Research Society, Montreal, 1981

108. Yamamoto, H., Tani, T., Mac Ewen, GD., Herman, R.: An evaluation of brain stem function as a prognostication of early idiopathic scoliosis. J Pediatr. Orthop. 2, 521, 1982.

109. Yarom, R. Carmy, O., Robin, GC., More, R.: Diffusion chamber cultures of muscle from patients with scoliosis. Hum. Pathol. 21/7, 623,1981 .

110. Yarom, R., Robin, GC.: Muscle pathology in idiopathic scoliosis. ISR. J. Mell. Sci 15/II, 917, 1979.

111. Yarom, R. Robin, GC.; Studies on spinal and peripheral muscles from patients with scoliosis. Spine, 4, 12, 1979.

112. Yarom, R., Robin, GC., Gorodetsky, R.; X-Ray fluorescence analysis of muscles in scoliosis. Spine, 3/", 142, 1978

113. Yarom, R. Wolf, E. Muhlrad, A., Robin, G.: Neuromuscular causes of idiopathic scoliosis. Ed. Scoliosis Research Soceity: Pathogenesis of idiopathic scoliosis. 153, 1984.

114. Zaleske, DJ., Ehrlich, MG. and Hall, JE.: Association of glicosaminoglycan depletion and degradative enzyme activity in scoliosis. Clin. Orthop., 148, 1980.

115. Zetterberg, C., Aniansson, A. Grimby, G.: Morphology of the paravertebral muscles in adolescent idiopathic scoliosis. Spine, 8/5, 457, 1983.

116. Zetterberg, C., Bjork, R., Ortengren, R. and Adersson, GBY. Electromyography of the paravertebral muscles in idiopathic scoliosis. Acta Orthop. Scand. 55, 304, 1984.

117. Zuck, T.: The role of spinal and abdominal muscles in the pathogenesis of scoliosis. J. Bone Joint Surg. 44B, 102, 1962. 